\title{
Application of Sweeping Jet Actuators on the NASA Hump Model and Comparison with CFDVAL2004 Experiments
}

\author{
Mehti Koklu* \\ NASA Langley Research Center, Hampton, VA 23681
}

\begin{abstract}
Flow separation control over a wall-mounted hump model was studied experimentally to assess the performance of sweeping jet actuators. Results were compared to that of the 2004 CFD validation experiment (CFDVAL2004), which examined flow separation control with steady suction and unsteady zero-net-mass-flow actuators. Comparisons were carried out at low and high amplitude excitations. In addition to the active flow control methods, a passive flow control method (i.e., vortex generator) was used to complement the dataset. Steady/unsteady surface pressure measurements and surface oilflow visualization were used in the performance assessment of the actuators. The results indicated that the sweeping jet actuators are more effective than the steady suction and unsteady zero-net-mass-flow actuators. For the same momentum coefficient, the sweeping jet actuators produced more flow acceleration upstream of separation, more pressure recovery downstream, and consistently a smaller separation bubble.
\end{abstract}

$\begin{array}{lll}A_{j}= & \text { total jet area } \\ A_{\infty}= & \text { projected model area } \\ c= & \text { hump chord length } \\ C_{\mu}= & \text { momentum coefficient } \\ C_{p}= & \text { pressure coefficient } \\ C_{p}{ }^{\prime}= & \text { fluctuating pressure coefficient } \\ Q= & \text { actuator flow rate } \\ \operatorname{Re}= & \text { Reynolds number based on chord } \\ s= & \text { model span } \\ U_{j}= & \text { jet velocity } \\ U_{\infty}= & \text { freestream velocity } \\ x, z= & \text { streamwise and spanwise directions }\end{array}$

Nomenclature

\section{Introduction}

Flow separation is the detachment of fluid from a surface, for example due to an adverse pressure gradient, and can be encountered in many engineering applications such as aircraft wings, high-lift systems, helicopter rotors, turbo machinery blades, diffusers, etc. Flow separation causes significant loss in the performance of a fluid system in the form of reduced lift, increased drag, reduced pressure recovery, etc. Flow separation control, or historically referred to as boundary layer control, involves manipulating the near wall fluid to delay, reduce, or sometimes completely prevent flow separation. Developing methods to control flow separation could help regain or improve the performance of the fluid systems. Various active and passive flow control concepts have been proposed to control flow separation including, but not limited to, steady suction, tangential blowing, passive micro-vortex generators (MVG), ${ }^{1}$ oscillatoryblowing valves, ${ }^{2}$ plasma actuators, ${ }^{3,4}$ zero-net-mass-flow actuators (ZNMF) ${ }^{5}$ and fluidic oscillators. ${ }^{6}$ Efficiency, complexity, and maintainability are key factors that prevent widespread utilization of these proven separation control techniques. ${ }^{7}$ In the context of efficiency, it is generally accepted that an unsteady excitation is much more effective than the steady flow control techniques to achieve prescribed performance improvement. ${ }^{2}$ Out of these various techniques, the fluidic oscillators have been shown to be simple, reliable, and efficient flow-control devices that can generate spatially and temporally oscillating (i.e., unsteady) jets without having any moving components.

\footnotetext{
*Aerospace Engineer, Flow Physics and Control Branch, MS 170, Member AIAA.
} 1 
Although there is a growing interest in fluidic oscillators (i.e., sweeping jet actuators) as a flow control device, there are only a few studies that investigated how well the sweeping jet (SWJ) actuators perform compared to the other flow control techniques. For example, the SWJ actuators were used to control flow separation on an adverse pressure gradient ramp ${ }^{8,9}$ and it was shown that they are more efficient than steady discrete blowing and steady vortex generating jets. The SWJ actuators were also compared to two dimensional slot blowing and pulsed jets on a highly separated transonic diffuser, and it was reported that the SWJ actuators and pulsed jets are more efficient than the steady two dimensional slot blowing, although both provided comparable performance. ${ }^{10}$

The objective of the present study is to assess the performance of the SWJ actuators as a flow separation control device. The wall-mounted hump model, which has been used as a benchmarking case for computational fluid dynamic (CFD) validations, was used as a test bed. The model was one of the case studies in the CFD Validation of Synthetic Jets and Turbulent Separation Control Workshop (CFDVAL2004) in 2004 and is well documented both experimentally ${ }^{11,12,7}$ and numerically ${ }^{13}$ over a wide range of Reynolds numbers $\left(0.4 \times 10^{6}<R e<26 \times 10^{6}\right)$. Using the data available in the literature, the performance of the SWJ actuators was compared to existing flow control techniques including steady suction and ZNMF actuators. The present study also provides a dataset complementary to the existing literature with different flow control techniques including MVGs and SWJ actuators.

\section{Experimental Setup}

The wind tunnel model is a wall-mounted hump originally designed by Glauert ${ }^{14}$ and later tested in low ${ }^{11-12}$ and high ${ }^{7}$ Reynolds number flows. The current model is the same model that was used by Greenblatt et al. ${ }^{11-12}$ in the same wind tunnel. The details about the model and the wind tunnel conditions can be found in Ref. [11]. Only a brief summary will be given for completeness. The schematic of the model in the wind tunnel is given in Fig. 1. The characteristic length is defined as the hump chord, which is $420 \mathrm{~mm}$. The original model had a two dimensional slot located at $x / \mathrm{c}$ $=0.65$ that was connected to an interior plenum spanning the entire model width $(s=584 \mathrm{~mm})$ between the forebody and ramp. In order to accommodate the sweeping jet actuators, the stainless steel slot-lip section was refabricated. The slot-lip section was divided into two sections, where the top section housed the surface static pressure ports, and the bottom section housed the sweeping jet actuator array. The actuator layer extended to the model ramp section such that it sealed the existing plenum on the model surface. The data reported in Ref. [11] was acquired while there was a suction manifold under the splitter plate. In our experimental configuration, the suction manifold was removed, and the plenum was sealed by a base plate to minimize the blockage.

The experiments were conducted in the NASA Langley Shear Flow Tunnel at a freestream Mach number of 0.1, which corresponded to $\mathrm{Re}=0.94 \times 10^{6}$ based on the hump chord. The experimental configuration was chosen to match to that of Ref. [11]. The model was mounted on a splitter plate between two endplates. Taking the model leading edge as $x=0$, the leading edge of the splitter plate was at $x=-1.935 \mathrm{~m}$, and the trailing edge of the splitter plate was at $x=1.129 \mathrm{~m}$. The boundary layer was tripped near the leading edge of the splitter plate using $20 \mathrm{~mm}$ thick (\#60) sand paper. Oilflow visualizations were performed prior to the wind tunnel tests to confirm that there was not a separation bubble at the leading edge of the splitter plate.

Surface static and dynamic pressures were acquired in this study. The model has 124 static pressure ports $(0.5$ $\mathrm{mm}$ orifice diameter) along the centerline and 16 spanwise pressure ports both on the forebody $(x / c=0.19)$ and on the ramp $(x / c=0.86)$ sections (Fig. 2). The static pressure ports were connected to electronically scanned pressure modules. Seventeen dynamic pressure ports were used to acquire fluctuating pressures in the separated flow region on the ramp section. Most of the dynamic pressure ports were aligned $25 \mathrm{~mm}(1 \mathrm{inch})$ off the centerline with the exception of first three ports between $x / c=0.69$ and $x / c=0.73$, which were aligned $38 \mathrm{~mm}$ (1.5 inches), $51 \mathrm{~mm}(2$ inches), and $63 \mathrm{~mm}$ ( 2.5 inches) off the centerline, respectively and the last two pressure ports that were located along the centerline (see port locations in Fig. 2). Miniature piezoresistive pressure transducers $( \pm 6.9 \mathrm{kPa}, 1 \mathrm{psig}$ ) were directly attached to the dynamic pressure ports.

Oilflow visualization was performed to map the surface flow patterns. The surface flow visualization was obtained using a mixture of kerosene, aviation oil, and fumed silica particles. Details of the flow visualization technique can be found in Ref. [8]. The mixture was applied to black contact paper mounted on the hump model and was moved under the effect of local shear stresses. Fluorescent pigment in the aviation oil glows under UV lighting and reveals the surface flow patterns.

The SWJ actuator array consisted of 17 actuators that spanned the entire model width (Fig. 3). The actuators were arranged in a lateral line pattern in which the middle actuator was located at the model centerline. The SWJ actuator spacing was $33 \mathrm{~mm}$. The SWJ actuator geometry (see Fig. 3 inset) was similar to the Mod 2 version of the actuator that was previously used in Ref. [15] with a throat dimension of $1 \mathrm{~mm}$ by $2 \mathrm{~mm}$. The actuator exits were placed at the same streamwise location as the original suction slot (i.e., $x / c=0.65$ ). The SWJ actuator array was 
fabricated using high-resolution stereolithography. The jet axis of the SWJ actuators pointed parallel to the freestream flow; however, the angle between the jet axis and local flow was approximately $20^{\circ}$ due to the surface curvature at this location. Each actuator in the array shared the same plenum. The flow rate to the actuator array was controlled by an electronic pressure regulator and monitored by a commercial flow meter. The SWJ actuator exits were not sealed externally during the experiments. The effect of actuator exits was also investigated (not shown here) but negligible difference was found in the static and dynamic pressure measurements. The negligible effect of actuator exit geometry was also reported in Ref. [11] with a continuous slot.

MVGs were also utilized to control flow separation on the hump model as passive flow control devices. MVGs were made out of $5 \mathrm{~mm}$ high and $19 \mathrm{~mm}$ long thin metal shim stock in a trapezoidal shape. Seventeen counter rotating MVG pairs were placed laterally across the span (Fig. 4). The spacing between the MVG pairs was $33 \mathrm{~mm}$ and the middle MVG pair was located at the model centerline similar to the SWJ actuators. The leading edges of the MVGs were $3 \mathrm{~mm}$ away from MVG symmetry line. MVGs were oriented at $\pm 23^{\circ}$ angles with respect to the direction of the freestream flow and placed near the suction peak $(x / c=0.48)$ in the streamwise direction to generate stronger streamwise vortices. ${ }^{1}$

\section{Results}

This section is divided into four subsections to present results associated with the flow over the NASA hump model. All of the experiments were conducted at a single freestream Mach number of 0.1 , which corresponded to $\operatorname{Re}=0.94$ $\times 10^{6}$. The first subsection describes the baseline flow over the hump model and compares it to the reference data. The first section also presents the effect of Reynolds number on the baseline flow. The second subsection describes the flow separation control with MVGs. The third subsection compares the SWJ actuators to an unsteady excitation technique (ZNMF actuators) at two different excitation amplitudes. Finally, the fourth subsection completes the dataset by comparing the SWJ actuators to a steady excitation technique (steady suction), again, at two different amplitudes.

\section{A. Baseline Separated Flow}

Before delving into the separated flow and its control, the boundary layer characteristics will be described and compared to the reference data. Figure 5 shows the boundary layer profile at the inflow location $(x / c=-2.14)$ and compares it to that of Ref. [11]. There is a noticeable difference in the boundary layer profile. The current boundary layer is substantially thinner and fuller than the reference boundary layer. The boundary layer thickness is approximately $19 \mathrm{~mm}$ (compared to $30.5 \mathrm{~mm}$ in Ref. [11]). Note that the analytical expression for a flat plate $\left(0.16 x / R_{x}{ }^{1 / 7}\right)$ predicts a boundary layer thickness of $20 \mathrm{~mm}$. The current momentum thickness is $2.14 \mathrm{~mm}$, which was $3.11 \mathrm{~mm}$ for the reference data. ${ }^{11}$ This represents a $30 \%$ lower Reynolds number based on momentum thickness (7200 vs. 4800). The reason behind the difference in the incoming boundary layer for the same experimental configuration is unknown; however, it is speculated that the suction manifold used in Ref. [11] introduced partial blockage to the flow under the splitter plate. This might shift the stagnation point under the splitter plate resulting in a separation bubble at the leading edge of the splitter plate. Although the separation bubble might be reattached to the splitter plate surface downstream, it thickened the boundary layer beyond its natural growth. In this study, the suction manifold was removed, and the oilflow visualization (not shown) was performed to check the existence of any separation bubble at the leading edge of the splitter plate. The shape factor at this location was calculated as 1.35 indicating a turbulent boundary layer approaching the hump model. The turbulent intensity at the inflow location was measured to be approximately $0.3 \%$.

The centerline static pressure distribution $\left(C_{p}\right)$ agrees well with the reference dataset in the majority of the domain (Fig. 6). The decelerated flow upstream of the hump model rapidly accelerates until $x / c=0.2$ due to the favorable pressure gradient, then the acceleration relaxes, and the pressure distribution reaches peak suction at $x / c=0.5$. The pressure discontinuity near the suction peak is due to a small step between the aluminum forebody and the stainless steel slot-lip section. ${ }^{11}$ The pressure recovery starts immediately after the suction peak and persists until flow separation at $x / c=0.66$. The current $C_{p}$ distribution is slightly lower (maximum deviation is less than 5\%) compared to the reference data in the separated region, where the flow above the separation bubble accelerates. Although the separated flow field was reported to be insensitive to the inflow conditions, ${ }^{7,11}$ this minor offset may be due to the substantial difference in the incoming boundary layers. A minor variation in the $C_{p}$ distribution, especially in the separated flow region $(0.69<x / c<0.92)$, was also reported in Ref. [16] when studying the effect of an incoming boundary layer at high Reynolds numbers. The fluctuating pressure distribution also agrees well with the reference dataset. Note that the current dataset is missing two unsteady pressure ports near the $C_{p}{ }^{\prime}$ peak. Since the reference data has all the fluctuating pressures and the current $C_{p}{ }^{\prime}$ distribution matches very well with the reference data for the 
rest of the points, no attempt was made to recover those missing ports. $C_{p}{ }^{\prime}$ usually peaks near (slightly upstream) the flow reattachment due to the intermittent nature of the reattachment process and the associated unsteady stagnation point. ${ }^{7}$ Although not precise, the $C_{p}{ }^{\prime}$ peak is a good indicator of the reattachment location.

Surface oilflow visualization for the baseline separated flow is presented in Fig. 7. The flow direction is from top to bottom. Two small corner vortices are observed downstream of the separation line at each side. A previously developed oilflow visualization technique enabled surface flow visualization for the entire model span without any excessive oil accumulation. As shown in this figure, the separation line is essentially two dimensional with the exception of the near-endplate regions. The oilflow visualization image was post processed to find the flow separation and reattachment locations. The separation location was found to be at $x / c=0.66$, similar to the reference dataset. The flow reattachment is fairly uniform around the centerline; however, the reattachment points move upstream closer to the corner vortex. Each corner vortex generates a reattachment node, where one can see oil movement in all directions. Similar lateral flow was also noted in Ref. [11]. The average reattachment location around the centerline is $x / c=1.15$ and $x / c=1.11$ near the endplate. The difference in the reattachment locations between the current and the reference data is approximately $17 \mathrm{~mm}(0.04 x / c)$.

It was shown previously that the Reynolds number has a negligible effect on the centerline $C_{p}$ distribution for $\operatorname{Re}$ $>0.5 \times 10^{6}$. This statement is confirmed in Fig. 8, where the centerline $C_{p}$ distribution is almost identical for three different Reynolds numbers. Insensitivity of the flow to the Reynolds number was linked to the elimination of laminarturbulent transition from the problem. ${ }^{16}$ Slightly higher pressures were observed near the flow reattachment as the Reynolds number increases. This is accompanied by slightly larger pressure fluctuations in the separated flow region. Spanwise pressure distributions at the forebody $(x / c=0.19)$ and the separated flow $(x / c=0.86)$ regions are given in Fig. 9 for various Reynolds numbers. As shown in this figure, the baseline separated flow over the hump model is essentially two dimensional with the exception of the near-endplate regions due to the corner vortices as shown in the surface oilflow visualization (Fig. 7).

\section{B. Flow Control with MVGs}

It is generally accepted that the MVGs generate streamwise vortices that increase the boundary-layer mixing and enhance the convective transport of the freestream momentum toward the near wall region. ${ }^{1}$ This in turn energizes the near-wall fluid momentum and enhances its resistance to flow separation. Before investigating the performance of the SWJ actuators, first the effect of MVG-generated streamwise vortices on the separated flow will be presented. This particular MVG arrangement (spacing, spanwise location, orientation, and number of MVGs) described previously and shown in Fig.4 was chosen to make a fair comparison with the SWJ actuators by producing the same number of streamwise vortices at the same spanwise locations.

Figure 10 presents the centerline $C_{p}$ distribution of the MVG flow control case and compares it to the baseline case. MVG location $(x / c=0.48)$ is described as a vertical dashed line in this figure. Four pressure ports near the suction peak are missing in the MVG case as they were blocked by the MVGs. Application of MVGs accelerates the flow upstream of separation and provides substantial pressure recovery compared to the baseline case. Note that the flow acceleration upstream of MVGs is minor due to the presence of MVGs but significant between the MVGs and flow separation. The magnitude and location of the suction peak increase from $C_{p}=-0.88$ (at $x / c=0.5$ ) to $C_{p}=-1$ (at $x / c=0.61$ ) providing a steeper pressure recovery as intended by the original model design. ${ }^{14}$ The dots in this figure represent the mean static pressures obtained by the unsteady pressure transducers that were $25 \mathrm{~mm}$ off-centerline (see port locations in Fig. 2). As shown in this figure, the off-centered static $C_{p}$ distribution matches very well with the centerline $C_{p}$ distribution indicating two dimensional mean flow near the centerline.

The $C_{p}{ }^{\prime}$ distribution in the separated flow region gets narrow, and the $C_{p}{ }^{\prime}$ peak moves upstream to $x / c=0.78$ for MVG flow control. This indicates a significantly shorter separation bubble compared to the baseline case. The magnitude of the $C_{p}{ }^{\prime}$ peak appears to be increased but the $C_{p}{ }^{\prime}$ peak for the baseline case may actually be higher. The limited spatial resolution of the unsteady pressure ports do not capture the $C_{p}{ }^{\prime}$ peak at $x / c=1.15$, where the reattachment location was obtained from the oilflow visualization (Fig. 7). Usually, the flow reattachment is slightly downstream of the $C_{p}{ }^{\prime}$ peak; therefore, there is a great possibility of higher $C_{p}{ }^{\prime}$ magnitudes between two dynamic pressure ports at $x / c=1.15$ and $x / c=1$. In addition, MVGs generate streamwise vortices, and due to the close proximity of the dynamic pressure ports to the MVGs, it is also possible that the higher values of $C_{p}{ }^{\prime}$ show the local effect of the streamwise vortices. This is consistent with the $C_{p}{ }^{\prime}$ discontinuity at the third unsteady pressure port. As explained in the experimental setup section (Fig. 2), the first three unsteady pressure ports were not aligned with the rest of the ports to capture flow three dimensionality near the actuator exits. While the $C_{p}{ }^{\prime}$ distribution of the baseline flow is uniform at the first four ports, the discontinuity in the $C_{p}{ }^{\prime}$ distribution for the MVG case indicates three dimensional flow structures. This is expected because several discrete streamwise vortices were introduced along the 
spanwise direction. Since the spacing between MVG pairs is $33 \mathrm{~mm}$, the first unsteady pressure port (38 $\mathrm{mm}$ off the centerline) is subjected to a common-flow-down counter rotating vortex; the second unsteady pressure port (51 mm off the centerline) is subjected to a common-flow-up counter rotating vortex; and finally the third unsteady pressure port (63 $\mathrm{mm}$ off the centerline) is subjected to common-flow-down vortices.

Surface oilflow visualization for the MVG flow control case is given in Fig. 11. Tunnel flow is from top to bottom. In an oilflow visualization, the streamwise vortices move oil material laterally in the rotation direction and lift it off the surface. The lines, where oil material is lifted off, are referred to as flow detachment lines. These flow detachment lines are clearly visible as dark lines downstream of each MVG in Fig.11 and denote the existence of streamwise vortices. The surface flow visualization begins immediately downstream of the MVGs and extends well beyond flow reattachment. The bottom border of this figure corresponds to the flow reattachment location for the baseline case. The streamwise vortices considerably reduce the effect of an adverse pressure gradient thereby reducing flow separation. The separation location appears to move slightly downstream $(x / c=0.67)$ by MVG flow control. Post processing of the oilflow visualization image reveals that the reattachment location moved approximately to $x / c$ $=0.75$, which is consistent with the movement of the $C_{p}{ }^{\prime}$ peak. Compared to the oilflow visualization of the baseline case (Fig. 7), the corner vortices appear to have a larger footprint in the streamwise direction.

Interaction of the streamwise vortices with the adverse pressure gradient on the hump model resulted in interesting flow structures as shown in the zoomed image of the MVG oilflow visualization (Fig. 12). The double lines at the top of the figure represent the orientation of MVGs relative to the flow visualization image. In this configuration, diverging MVGs generate common-flow-down counter rotating vortices; whereas converging MVG pairs generate common-flow-up counter rotating vortices. Interestingly, the flow detachment lines bend inward symmetrically to the axis of diverging MVGs near flow separation, and oil accumulations are observed downstream of each diverging MVG. This is counterintuitive because usually one can see oil accumulation downstream of common-flow-up vortices (i.e., between converging MVGs) as they locally thicken the boundary layer further upstream. In addition, the common-flow-up vortices transport oil material toward their symmetry axis due to their rotation direction and have been shown to generate small pockets of recirculation regions both numerically ${ }^{17}$ and experimentally. ${ }^{18}$ The complex flow at this region should be investigated further maybe using an off body measurement technique such as particle image velocimetry. This figure also explains the discontinuities in the $C_{p}{ }^{\prime}$ distribution between the first four unsteady pressure ports. The dots in this figure represent the relative location (not to scale) of the first four unsteady pressure ports. As shown in this figure, these ports are inside the separation bubble and are exposed to different complex flow structures. The flow reattachment is no longer a line, instead it involves numerous reattachment nodes and saddle points across the span. Note that although the zoomed-in image shows that these reattachment nodes/saddle points are somewhat downstream of diverging MVGs, they are not necessarily aligned with the MVGs.

\section{Comparison of SWJ Actuators with ZNMF Actuators}

Experiments with zero-net-mass-flow (ZNMF) actuators to control flow separation on the same hump model were performed as part of the CFD validation workshop, and the experimental results were previously reported in Ref. [12]. The ZNMF actuators were voice-coil based actuator modules that produced unsteady (synthetic) jets out of a two dimensional slot. The test case selected for the comparison reported to produce a peak jet velocity of $26.6 \mathrm{~m} / \mathrm{s}$ at 138.5 $\mathrm{Hz}$ actuation. ${ }^{12}$ This actuation corresponds to a momentum coefficient of $C_{\mu}=0.11 \%$, whose definition is given as $C_{\mu}=2 A_{j} U_{j}^{2} / A_{\infty} U_{\infty}^{2}$, where $A_{\infty}$ is the projected model area $\left(420 \times 584 \mathrm{~mm}^{2}\right), A_{j}$ is total jet area $\left(0.78 \times 584 \mathrm{~mm}^{2}\right)$, and $U_{j}$ is the jet exit velocity (in $\mathrm{m} / \mathrm{s}$ ). Since the ZNMF actuators were assumed to produce zero net mass flow during one operation cycle, $U_{j}$ is due to the oscillatory component of the ZNMF excitation as explained in Ref. [12]. For the SWJ actuators, $A_{j}$ is the number of actuators times the actuator throat area $\left(17 \times 1 \times 2 \mathrm{~mm}^{2}\right)$, and $U_{j}$ is the jet velocity at the throat, which was estimated from the flow rate and the total jet area $\left(U_{j}=Q / A_{j}\right)$. Note that the actuator throat is slightly upstream of the actuator exit as shown in Fig. 3. Due to the discrete blowing, the reduction in the jet area resulted in an increased jet velocity for the SWJ actuators $\left(U_{j}=69 \mathrm{~m} / \mathrm{s}\right)$. For this particular momentum coefficient $\left(C_{\mu}=0.11 \%\right)$, the flow rate to the SWJ actuator array was $Q=2.4 \mathrm{~L} / \mathrm{s}$, and the actuator oscillation frequency was $550 \mathrm{~Hz}$, as measured by an unsteady pressure port close to the actuator exit. Since the flow rate to the actuator plenum (not to the individual actuators) was monitored during the experiment, the uniformity of flow distribution to each SWJ actuator was checked prior to the wind tunnel test. The flow uniformity was verified by measuring and comparing the frequency of individual actuators in the actuator array on a bench top. Since the frequency of an SWJ actuator is usually proportional to the flow rate, the uniformity of the measured frequencies also implies the uniformity of the actuator flow rate and hence the actuator exit velocity. The bench top test with the SWJ actuators showed that the actuator frequencies, and hence the flow rates, deviate a maximum of $2.5 \%$. 
Figure 13 compares the centerline $C_{p}$ distribution of the SWJ actuators to that of the ZNMF actuators at the same momentum coefficient $\left(C_{\mu}=0.11 \%\right)$. The location of the actuator exit $(x / c=0.65)$ is described as a vertical dashed line in this figure (also in the rest of the pressure distribution figures). The pressure distribution of the baseline and MVG flow control cases are also given for comparison. As shown in this figure, both active flow control (AFC) methods produced substantial pressure recovery in the separated flow region. They both have similar trends but the efficiency of the SWJ actuators is evident when compared to the ZNMF actuators. The SWJ actuators produced substantial flow acceleration upstream of the actuation that increased the suction peak from -0.88 to -0.95 ; whereas the ZNMF actuators increased the suction peak only to -0.89 . Both AFC methods appear to promote flow separation immediately downstream of the actuators as indicated by the sudden decrease in pressure. The $C_{p}$ distribution in this region is shifted up - indicating flow reacceleration over the separation bubble - and only at $x / c=0.75(x / c=0.77$ for ZNMF) the mixing rate is high enough to start decelerating the flow and recovering the pressures. The length of the flow reacceleration $\left(C_{p}\right.$ decrease) region immediately downstream of the actuators is also related to the separation bubble size and implies that the SWJ actuators are more effective than the ZNMF actuators. Although the optimum ZNMF actuation frequency $(250 \mathrm{~Hz})$ at the same momentum coefficient provided slightly better performance than the $138.5 \mathrm{~Hz}$ case considered here, ${ }^{12}$ it is still outperformed by the SWJ actuators. The passive flow control with MVGs provided better control authority than either of the AFC methods at this particular momentum coefficient.

The fluctuating pressures $\left(C_{p}{ }^{\prime}\right)$ are displayed in the lower right corner of this figure for all four cases (Fig. 13). The $C_{p}{ }^{\prime}$ distributions appear to have comparable magnitudes, whereas some slight variations are observed for different flow control methods. The $C_{p}{ }^{\prime}$ peak pressures move upstream due to the shorter separation bubble. Although the baseline and the MVG control show distinct changes between $x / c=0.8$ and $x / c=1.2$, the $C_{p}{ }^{\prime}$ distributions for the AFC methods are more broad, and it is difficult to determine the peak $C_{p}{ }^{\prime}$ location as an indication of reattachment location. The effect of two dimensional versus discrete actuation is also presented in this figure. While the two dimensional slot actuation with the ZNMF actuators provided smooth data for the first four unsteady pressure ports, the discrete actuation with the SWJ actuators resulted in discontinuities in the first four pressure ports similar to those observed in the $C_{p}{ }^{\prime}$ distribution of the MVG flow control case. These discontinuities indicate the presence of unsteady flow structures near the actuator exits for MVG and SWJ flow control cases.

Figure 14 shows the surface oilflow visualization for the SWJ actuator case with $C_{\mu}=0.11 \%$. The SWJ actuator array is also shown on top of the figure. Unfortunately, no oilflow visualization was provided in Ref. [12] for the ZNMF flow control case so detailed comparisons cannot be made. Therefore, the oilflow visualization for the SWJ actuator will be used to explain the effect of the SWJ actuators on flow separation. The flow visualization clearly shows the separation bubble and the reattachment location. The flow reattachment appears to be at $x / c=0.96$ near $z / s$ $=0$; however, it moves upstream to $x / c=0.92$ away from the centerline. Note that the reattachment point for the ZNMF actuators was reported as $x / c=0.98$. The reversed flow is still strong and able to transport oil material upstream even though the size of the separation bubble is reduced almost $40 \%$ compared to the baseline case. The lateral flow at the reattachment line is also visible in the flow visualization image forming a saddle point-like reattachment near the center. Each side of the figure shows two small reattachment nodes and a corresponding saddle point in between, which is similar to the flow visualization results for the MVG flow control case (Fig. 12). The flow visualization image shows oil accumulation downstream of the actuators near $x / c=0.75$. However, it is not clear if this is a separation location because oil material also moves under the effects of gravity, shear stress due to reversed flow, and the jet flow. Local oil accumulations are also observed at some of the actuator exits.

Another comparison between the ZNMF and the SWJ actuators was made at a higher excitation amplitude. The maximum reported momentum coefficient in Ref. [12] for the ZNMF actuators is $C_{\mu}=0.35 \%$ with the same $138.5 \mathrm{~Hz}$ actuation frequency. To obtain the same momentum coefficient, the flow rate to the SWJ actuators was increased to $Q=4.25 \mathrm{~L} / \mathrm{s}$, which resulted in a jet velocity of $U_{j}=120 \mathrm{~m} / \mathrm{s}$. At this flow rate, the oscillation frequency of the SWJ actuators was $875 \mathrm{~Hz}$. As shown in Fig. 15, increasing the excitation amplitude increased the flow acceleration upstream of actuators for both AFC methods. The ZNMF actuators produced a different pressure distribution in the pressure recovery region, where the high amplitude excitation caused less flow reacceleration (pressure decrease) immediately downstream of the actuators compared to the low amplitude excitation $\left(C_{\mu}=0.11 \%\right.$, Fig. 13). This was explained by Greenblatt ${ }^{12}$ as the effect of actuators being characterized differently for low and high amplitude excitations. Although the low amplitude excitation reduces the separation bubble as demonstrated by the pressure recovery as well as upstream $C_{p}{ }^{\prime}$ peak (Fig. 13), the circulation of the separation bubble is intensified as evidenced by the level of flow reacceleration ( $C_{p}$ decrease) over the separation bubble. However, for the excitation with $C_{\mu}=0.35 \%$, the level of low pressure is similar to the baseline case meaning that the excitation does not increase the strength of the bubble compared to the baseline case. The length of the flow reacceleration region immediately downstream of the actuators reduces considerably from $x / c=0.9$ to $x / c=0.75$ indicating a shorter separation bubble compared to the 
baseline case. Application of the SWJ actuators with the same amplitude increases the suction peak substantially to $C_{p}=-1.14$. In the pressure recovery region, the SWJ actuator performance is more than that of the ZNMF and MVG actuators reaching to positive $C_{p}$ values downstream. We do not observe any pressure decrease (flow reacceleration) due to the excitation immediately downstream of the actuators. In fact, we observe higher pressures compared to the baseline or ZNMF cases. There is a slight variation in the pressure distribution near $x / c=0.7$ possibly indicating a thin flow separation bubble.

Slightly higher pressure fluctuations are observed for the high amplitude excitations for both AFC methods (lower right of Fig. 15). The $C_{p}{ }^{\prime}$ peak (i.e., reattachment location) for the SWJ actuators is further upstream $(x / c=0.78)$ than the ZNMF actuators $(x / c=0.87)$ indicating a shorter separation bubble. The $C_{p}{ }^{\prime}$ distribution of the SWJ case is very similar to that of MVGs, although the SWJ actuators provided a higher suction peak and more pressure recovery. Interestingly, the three dimensional flow structures that were previously observed in the MVG and low amplitude SWJ cases are not present as evidenced by the $C_{p}{ }^{\prime}$ values at the first three unsteady pressure ports.

\section{Comparison of SWJ Actuators with Steady Suction}

Flow control with steady suction was reported in Ref. [11] together with the baseline case as part of the CFD validation workshop. Steady suction was applied from a two dimensional slot with a nominal slot width of $0.78 \mathrm{~mm}$ at the same Reynolds and Mach numbers. Although different suction rates were applied, the test case reported had a momentum coefficient of $0.24 \%$. As stated in Ref. [11], this suction rate was not enough to eliminate flow separation. The flow control with steady suction was able to maintain attached flow over the hump model with $C_{\mu}=2.6 \%$ and $C_{\mu}=4 \%$ for low $^{11}$ and high ${ }^{16}$ Reynolds numbers, respectively. For this particular momentum coefficient $\left(C_{\mu}=0.24 \%\right)$, the flow rate from the suction unit was $Q=12.4 \mathrm{~L} / \mathrm{s}$, which generated a suction jet velocity of $U_{j}=28 \mathrm{~m} / \mathrm{s}$ at the slot. In order to keep the same momentum coefficient for the SWJ actuators, the flow rate to the actuators was set to $Q=3.5 \mathrm{~L} / \mathrm{s}$, which generated $U_{j}=100 \mathrm{~m} / \mathrm{s}$ jet velocity and $753 \mathrm{~Hz}$ oscillation frequency.

The centerline pressure distribution is given in Fig. 16 for the momentum coefficient of $C_{\mu}=0.24 \%$. Flow control by means of steady suction increases the suction pressure (flow acceleration) upstream of the slot. ${ }^{11}$ As shown, there is a substantial pressure recovery compared to the baseline case. The existence of flow separation is evident from the pressure distribution, where a plateau is seen near $x / c=0.7$. The steady suction increases the pressure immediately downstream of the slot, meaning it also reduces the strength of the separation bubble. Application of SWJ actuators with the same momentum coefficient $\left(C_{\mu}=0.24 \%\right)$ increases the suction pressures upstream of the actuators. The pressure distribution matches very well with that of the steady suction case indicating both AFC techniques provided similar flow acceleration. The SWJ actuators do not improve the pressure recovery immediately downstream of the exit (until $x / c=0.73$ ) but provide slightly lower pressures compared to the baseline case. This implies that this level of SWJ application slightly intensifies the separation bubble although reducing its size. Comparison of Figs. 13, 15, and16 shows the effect of momentum coefficient for the SWJ actuators, where a gradual increase in the momentum coefficient proportionally increases the upstream suction peak and downstream pressure recovery. Consistently, the separation bubble slightly intensifies for low amplitude excitations $\left(C_{\mu}=0.11 \%\right.$, Fig. 13 and $C_{\mu}=0.24 \%$, Fig. 16) but weakens as the momentum coefficient increases. The $C_{p}$ distribution of the SWJ actuators starts to increase after $x / c$ $=0.73$, crosses the $C_{p}$ distribution of the steady suction, and provides more pressure recovery than that of the steady suction case. Considering the $60 \%$ reduction in the mass flow requirement, this level of control clearly shows the superiority of the SWJ actuators. Interestingly, the $C_{p}$ distribution of the SWJ actuation is similar to that of the MVG case in the pressure recovery region indicating both flow control methods have a similar level of flow control authority on the separated flow.

Fluctuating pressures show that the application of the SWJ actuators reduces the flow separation. The $C_{p}{ }^{\prime}$ peak moves further upstream to $x / c=0.78$ compared to $x / c=0.9$ for steady suction. The magnitude of the $C_{p}{ }^{\prime}$ distribution also increases compared to the steady suction due to the highly unsteady jet. The level and location of the $C_{p}{ }^{\prime}$ peak are similar to that of the MVG case, which implies similar separation bubble characteristics. Downstream of reattachment, the $C_{p}$ ' distribution of the SWJ actuators deviates from that of the MVGs. This can be explained as the generated streamwise vortices being diffused at a faster pace. The diffuse rate of the flow structures can be related to the excitation amplitude of the actuators. Comparing the $C_{p}{ }^{\prime}$ distributions of different excitation amplitudes (Figs.13, 15, and 16), we noticed that the pressure fluctuations downstream of reattachment gradually reduce with the momentum coefficient and eventually reach a similar level to that of the MVG case. Contrary to the baseline and steady suction cases, the $C_{p}{ }^{\prime}$ distribution of the SWJ actuators shows slight local three dimensionality as illustrated by the first three unsteady ports. While the variation between the first three ports is negligible for the baseline and steady suction control cases, it is prominent for the SWJ and MVG flow control cases. This is because steady suction was applied uniformly from a two dimensional slot, whereas the SWJ (and MVG) flow control was applied at discrete 
locations. Again comparing Figs. 13, 15, and 16 reveals that the three dimensionality is inversely proportional to the excitation amplitude, where the three dimensionality gradually decreases as the flow control authority increases.

Surface oilflow visualization for the SWJ actuators at $C_{\mu}=0.24 \%$ is presented in Fig. 17. Although the attached flow downstream is visible in the form of oil streaks, the reattachment location is not visually clear due to low shear stress. Post processing the flow visualization image reveals that the reattachment location is near $x / c=0.85$. Note that the reattachment point for the steady suction case was reported as $x / c=0.94$. This corresponds to a $40 \mathrm{~mm}$ shorter separation bubble compared to the steady suction case. The signature of reversed flow (oil streaks) is visible locally but it is not as evident as that of Fig. 14, where the level of flow control was lower, and hence the separation bubble was stronger. Globally, flow reattachment appears to be two dimensional. The separation location is not very clear due to the complex flow near the actuator exits.

The comparison of the SWJ actuators with steady suction at high excitation amplitude $\left(C_{\mu}=0.47 \%\right)$ is presented in Fig. 18. To obtain the same momentum coefficient, the flow rate to the SWJ actuators was increased to $Q=4.87$ $\mathrm{L} / \mathrm{s}$, which resulted in a jet velocity of $U_{j}=139 \mathrm{~m} / \mathrm{s}$. At this flow rate, the oscillation frequency of the SWJ actuators was $953 \mathrm{~Hz}$. The centerline pressure distribution shows a trend similar to what we saw in Figs. 13, 15, and 16 that is, increasing the momentum coefficient gradually increases the actuator's performance for both AFC methods. The superior performance of the SWJ actuators at higher actuation amplitude is evident over the entire $C_{p}$ distribution as they produce higher upstream suction pressures and more pressure recovery downstream. The suction peak increases beyond $C_{p}=-1.2$. The flow control with steady suction provided the same level of pressure recovery immediately downstream of actuators as that of the $C_{\mu}=0.24 \%$ case indicating similar extent of the separation bubble. The steady suction only resulted in a minor increase in the pressure recovery, although the excitation amplitude is doubled compared to the $C_{\mu}=0.24 \%$ case. This unquestionably indicates the loss of efficiency of steady suction for high excitation amplitude. These observations are also consistent with the $C_{p}{ }^{\prime}$ distributions of the low $\left(C_{\mu}=0.24 \%\right)$ and high $\left(C_{\mu}=0.47 \%\right)$ amplitude suction cases. The level and location of the $C_{p}{ }^{\prime}$ peak varied slightly, again indicating a separation bubble with similar size and strength. However, doubling the excitation amplitude of the SWJ actuators proportionally increased the pressure recovery. We do not observe any low pressure regions immediately downstream of the actuators, possibly due to the elimination of separation bubble. The $C_{p}{ }^{\prime}$ peak moves close to the actuator exits $(x / c=0.71)$, which may show the effect of unsteady jets rather than the flow reattachment.

Figure 19 illustrates the effect of SWJ flow control on the spanwise pressure distributions at $x / c=0.86$ for various excitation amplitudes. The figure also shows the spanwise $C_{p}$ distribution for baseline and MVG flow control cases for comparison. As shown in this figure, some slight deviations are observed in the spanwise $C_{p}$ distribution possibly due to discrete blowing rather than two dimensional actuation via a slot. These deviations are not correlated with the excitation amplitude nor biased toward one side of model. The spanwise $C_{p}$ distribution denotes that the corner vortices appear to be amplified by the increased flow control. This was not the case in the reference AFC dataset with the ZNMF actuator and steady suction because both of these AFC methods were applied from a two dimensional slot that spanned the entire model width. In the present study with SWJ actuators as well as MVGs, the excitation was applied from discrete locations, and there is a spacing of $28 \mathrm{~mm}$ between the endplate and the nearest actuators. Therefore, while the corner vortices were somewhat controlled in Refs. [11-12], they were not affected as much in the current case due to spanwise distance to actuators. In addition, the SWJ flow control appears to amplify these corner vortices because increasing the flow control also accelerates the flow and hence the rotation of the corner vortices.

\section{Conclusion}

An experimental study was performed to assess the performance of the sweeping jet (SWJ) actuators in controlling flow separation. The NASA hump model, which has been used as a benchmarking case for CFD validations and is well documented over a wide range of Reynolds numbers, was used as a test bed. Using the data available in the literature, the performance of the SWJ actuators was compared to the existing steady and unsteady flow control techniques including steady suction and zero-net-mass-flow (ZNMF) actuators. In addition to the performance assessment, the present study also provided a dataset complementary to the existing reference dataset for different flow control techniques including micro vortex generators (MVGs) and the SWJ actuators. Steady/unsteady surface pressure measurements and surface oilflow visualizations were used in the performance assessment of the SWJ actuators.

Prior to the active flow control experiments, the baseline separated flow and its control with MVGs were investigated. Boundary layer measurements at the inflow location showed that the current boundary layer is much thinner and fuller than the reference boundary layer and is close to that of the analytical expression. The discrepancy in the boundary layer profile was attributed to the removal of the suction manifold that caused partial blockage to the flow under the splitter plate in the reference dataset. Although having different inflow conditions, very good 
agreement was obtained in the pressure measurements with the reference dataset. This confirms the insensitivity of separated flow over the hump model to the inflow conditions such as upstream boundary layer thickness. A surface oilflow visualization, which was not provided in the reference dataset, revealed two dimensional flow separation and a uniform but slightly three dimensional flow reattachment line. The reattachment location obtained by oilflow visualization near the centerline indicates a $0.04 \mathrm{x} / \mathrm{c}$ longer separation bubble than the reference data. Introducing streamwise vortices using MVGs was very effective in controlling separation. Flow separation control with MVGs provided higher suction pressures upstream of separation, healthier pressure recovery downstream, and a substantially smaller separation bubble.

The performance of the SWJ actuators was first compared to an unsteady excitation (ZNMF actuators). For low amplitude excitations, both ZNMF and SWJ actuators have similar trends but the effect of SWJ actuators is more pronounced compared to the ZNMF actuators by providing higher suction pressures upstream of actuators and more pressure recovery downstream. Oilflow visualization and fluctuation pressures showed that the separation bubble for the SWJ actuators is shorter than what was reported for the ZNMF actuators. Although increasing the excitation amplitude increased the efficiency of both actuators, the SWJ actuators provided higher suction pressures and more pressure recovery downstream compared to the ZNMF actuators.

Another performance assessment was conducted by comparing the effect of the SWJ actuators with steady suction. For low amplitude excitations, where flow separation still exists, the SWJ actuators and steady suction produced similar flow acceleration upstream of separation, but different pressure recovery characteristics downstream. The steady suction was shown to be more effective in the vicinity of actuators providing a rapid pressure recovery as intended by the original hump design. On the other hand, the SWJ actuators slightly reduced the pressure immediately downstream of the actuators indicating flow acceleration, and hence a stronger separation bubble. However, the SWJ actuators provided even more pressure recovery further downstream. The reattachment location for the SWJ actuators using oilflow visualization indicated an almost $0.1 \mathrm{x} / \mathrm{c}$ shorter separation bubble compared to that of the steady suction case. For high amplitude excitations, the steady suction provided the same level of flow control authority in the vicinity of actuators and slightly better pressure recovery further downstream despite doubling the excitation amplitude. This implies the loss of efficiency of the steady suction as the excitation amplitude increases. On the other hand, the effect of the SWJ actuators proportionally increased with the excitation amplitude providing more flow acceleration upstream of separation and more pressure recovery downstream. These characteristics, coupled with the lower mass flow requirements, clearly substantiate the superior performance of the SWJ actuators in this application.

\section{Acknowledgments}

The author would like to thank the NASA Advanced Air Transport Technology Project for funding this research and the following individuals for their support: Catherine McGinley, Luther Jenkins, Latunia Melton, John Lin, and Charlie Debro.

\section{References}

${ }^{1}$ Lin, J.C., "Review of Research on Low-Profile Vortex Generators to Control Boundary-Layer Separation," Progress in Aerospace Sciences, Vol. 38, No. 4, 2002, pp. 389-420.

${ }^{2}$ Greenblatt, D. and Wygnanski, I. J., "The Control of Flow Separation by Periodic Excitation," Progress in Aerospace Sciences, Vol. 36, No.7, 2000, pp. 487-545.

${ }^{3}$ Roth, J. R., Sherman, D. M., and Wilkinson, S. P., "Electrohydrodynamic Flow Control with a Glow-Discharge Surface Plasma," AIAA Journal, Vol. 38, No. 7, 2000, pp. 1166-1172.

${ }^{4}$ Enloe, C. L., McLaughlin, T. E., VanDyken, R. D., Kachner, K. D., Jumper, E. J., Corke, T. C., Post, M., and Haddad, O., "Mechanisms and Responses of a Single Dielectric Barrier Plasma Actuator: Geometric Effects," AIAA Journal, Vol. 42, No. 3, 2004, pp. 595-604.

${ }_{5}^{5}$ Glezer, A. and Amitay, M., "Synthetic Jets" Annual Review of Fluid Mechanics, Vol. 34, 2002, pp. 503-529.

${ }^{6}$ Viets H. "Flip-flop jet nozzle", AIAA Journal, Vol. 13, 1975, pp.1375-1379.

${ }^{7}$ Seifert, A. and Pack, L. G., "Active Flow Separation Control on Wall-Mounted Hump at High Reynolds Numbers, "AIAA Journal, Vol. 40,No. 7, 2002, pp. 1363-1372.

${ }^{8}$ Koklu M., and Owens, L. R., "Comparison of Sweeping Jet Actuators with Different Flow-Control Techniques for FlowSeparation Control", AIAA Journal, Vol. 55, No. 3 (2017), pp. 848-860.

${ }^{9}$ Koklu, M., "The Effects of Sweeping Jet Actuator Parameters on Flow Separation Control," AIAA Paper 2015-2485, 2015.

${ }^{10}$ Gartner, J., and Amitay, M., "Flow Control in a Diffuser at Transonic Conditions" AIAA paper 2015-2484, June 2015.

${ }^{11}$ Greenblatt, D., Paschal, K. B., Yao, C. S., Harris, J., Schaeffler, N. W., and Washburn, A. E., "Experimental Investigation of Separation Control Part 1: Baseline and Steady Suction," AIAA Journal, Vol. 44, No. 12, 2006, pp. 2820-2830.

9

American Institute of Aeronautics and Astronautics 
${ }^{12}$ Greenblatt, D., Paschal, K., Yao, C.-S., and Harris, J., "Experimental Investigation of Separation Control Part 2: Zero MassEfflux Oscillatory Blowing," AIAA Journal, Vol. 44, No. 12, 2006b, pp. 2831-2845.

${ }^{13}$ Rumsey, C. L., Gatski, T, B., Sellers, W, L., Vatsa, V, N., and Viken, S. A., "Summary of the 2004 CFD Validation Workshop on Synthetic Jets and Turbulent Separation Control," AIAA Paper 2004-2217, 2004.

${ }^{14}$ Glauert, M. B., "The Design of Suction Aerofoils with a Very Large $C L$-Range," Aeronautical Research Council, R\&M 2111, Nov. 1945.

${ }^{15}$ Melton, L. P., Koklu, M., Andino, M., Lin, J. C., and Edelman L. M.," Sweeping Jet Optimization Studies”, AIAA paper 2016-4233, June 2016.

${ }^{16}$ Seifert, A., and Pack, L. G., “Active Control of Separated Flows on Generic Configurations at High Reynolds Numbers," AIAA Paper 1999-3403, June 1999.

${ }^{17}$ König, B., Fares, E., and Nölting, S., "Fully-Resolved Lattice-Boltzmann Simulation of Vane-Type Vortex Generators," AIAA Paper 2014-2795, June 2014.

${ }^{18}$ Lin, J., Howard, F., and Selby, G., "Small-Submerged Vortex Generators for a Turbulent Flow Separation Control," Journal of Spacecraft and Rockets, Vol. 27, No. 5, 1990, pp. 503-507. 


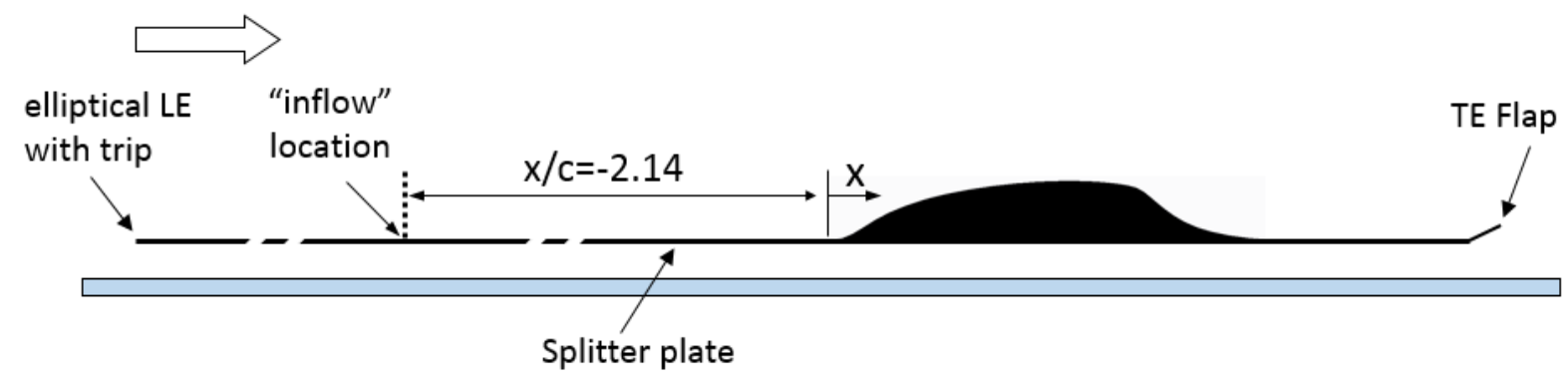

Figure 1. Schematic of the hump model in the wind tunnel.

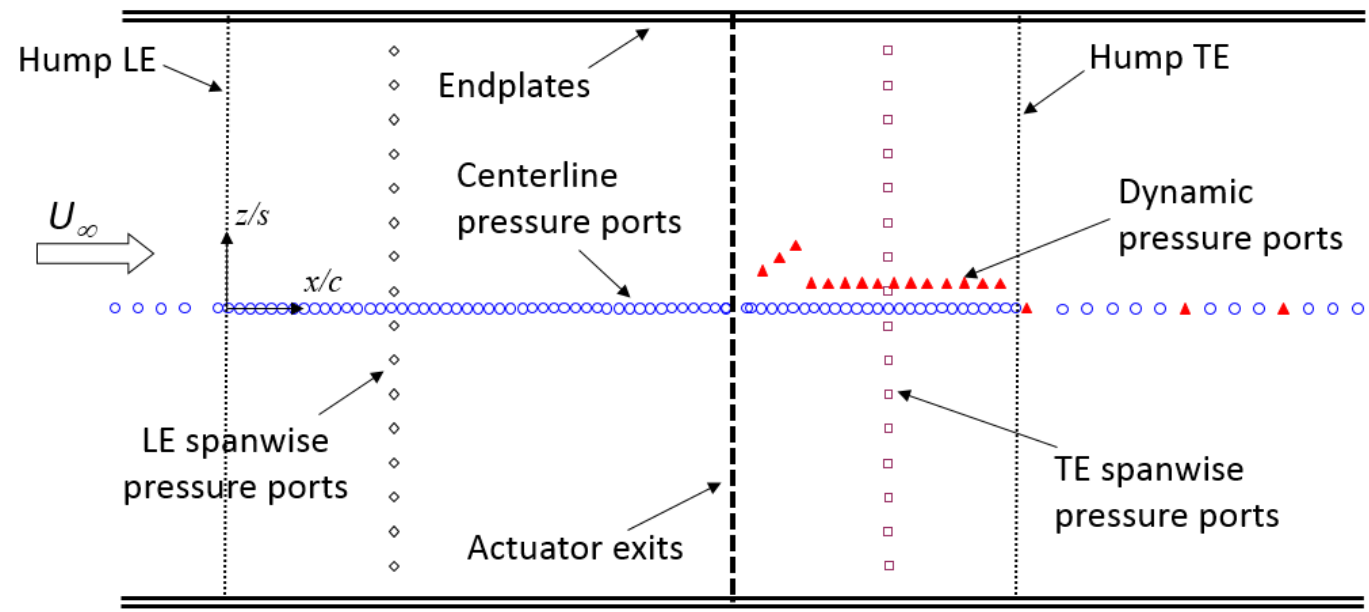

Figure 2. Surface static and dynamic port locations relative to the hump model.

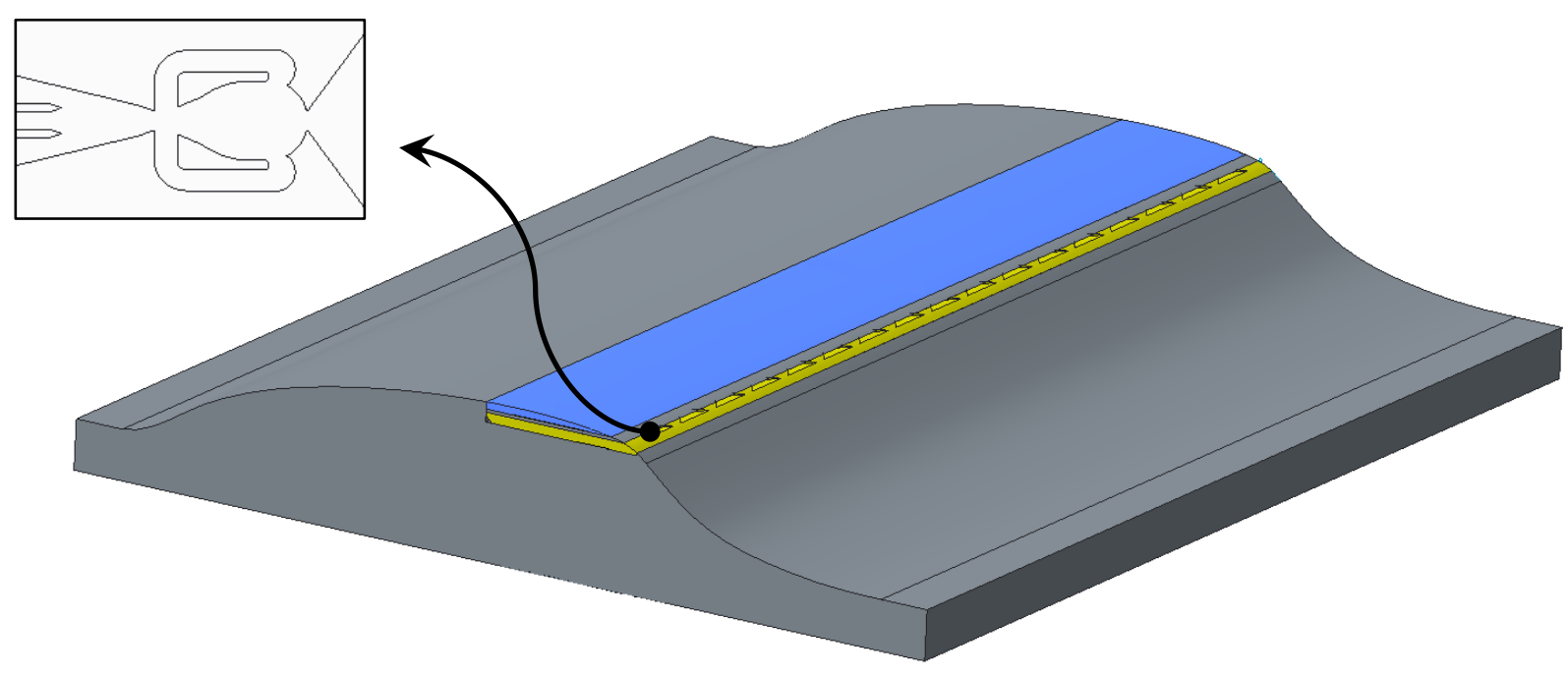

Figure 3. CAD rendering of the hump model (inset shows the SWJ actuator geometry). 


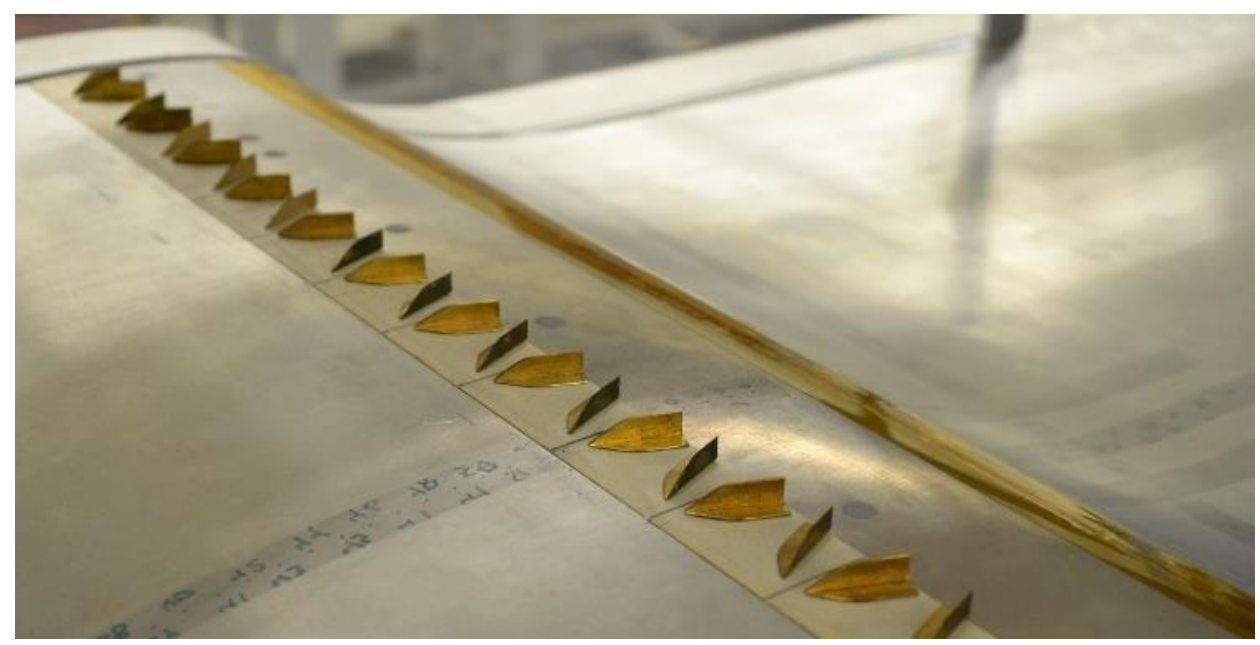

Figure 4. Photograph of MVGs installed on the hump model.

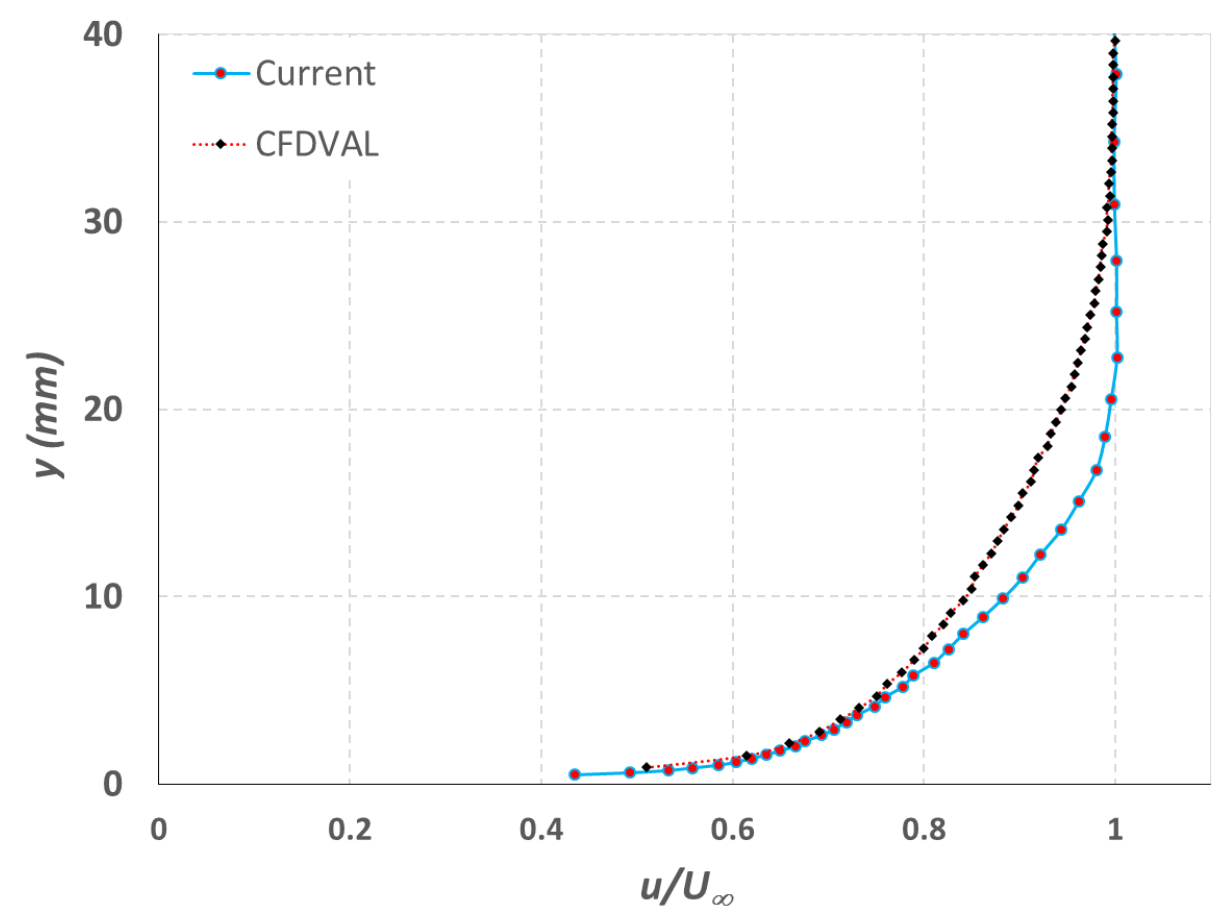

Figure 5. Comparison of boundary layer profile at the inflow location with the reference data. ${ }^{11}$ 


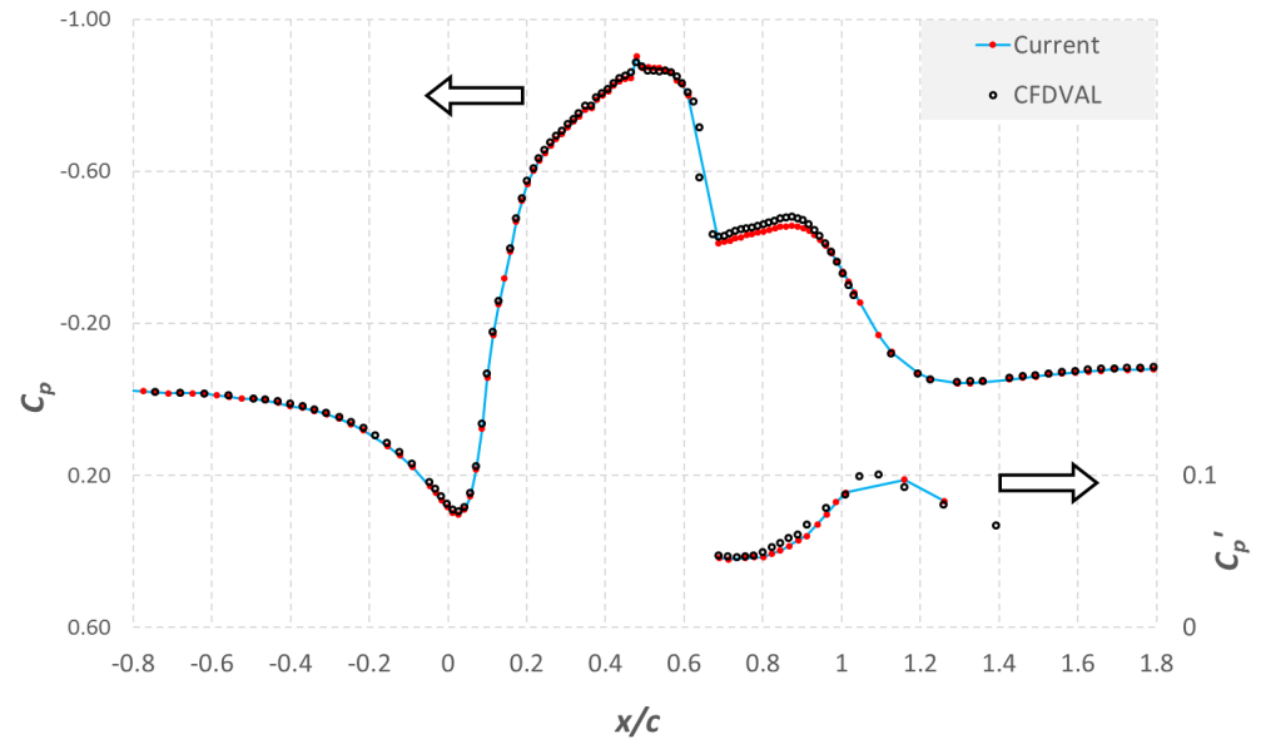

Figure 6. Comparison of the surface static and dynamic pressures with the reference data. ${ }^{11}$

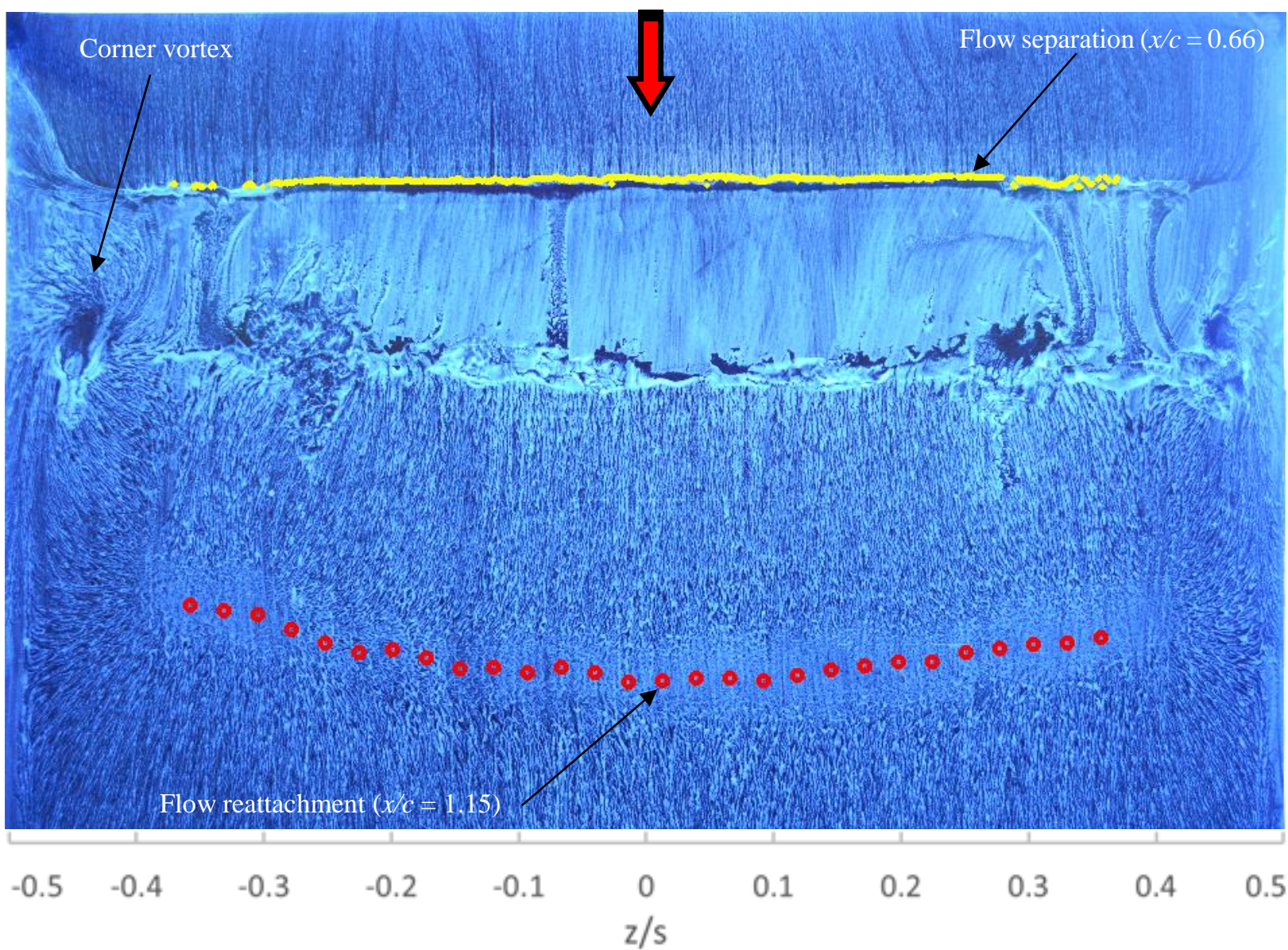

Figure 7. Surface oilflow visualization of the baseline flow showing flow separation and reattachment. 


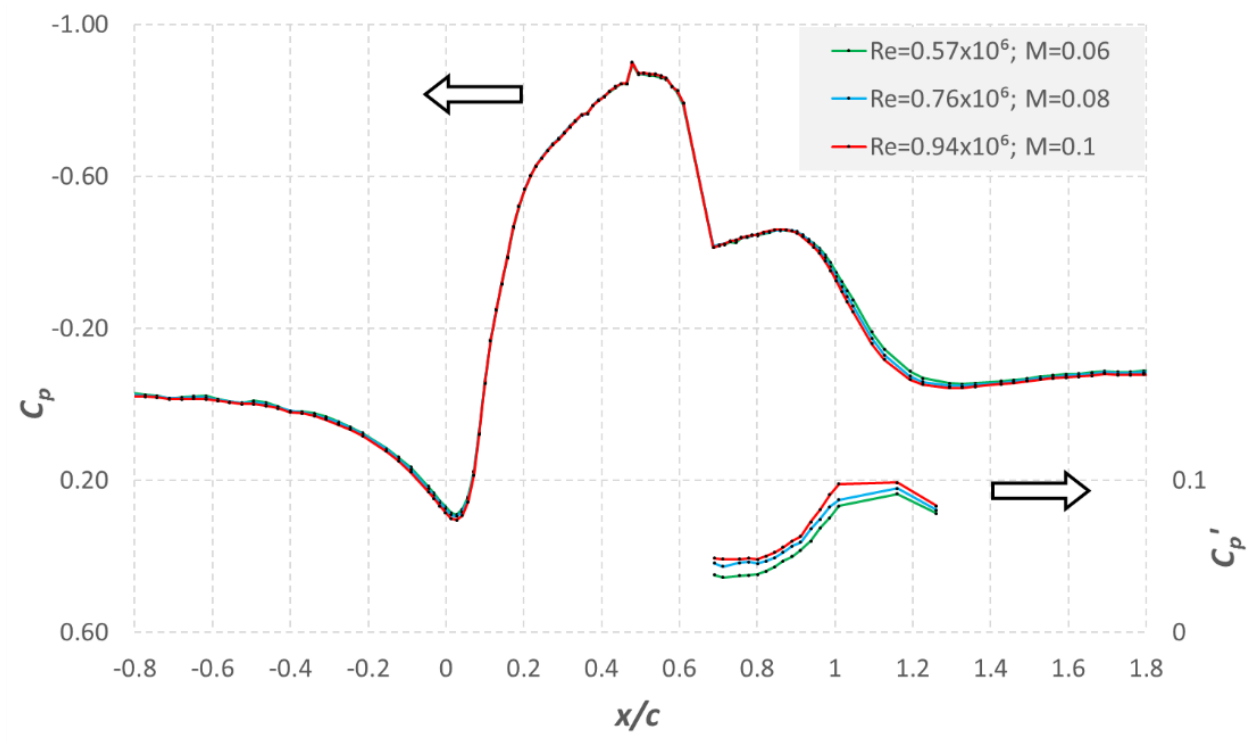

Figure 8. The effect of Reynolds number on the surface pressure distribution for the baseline case.

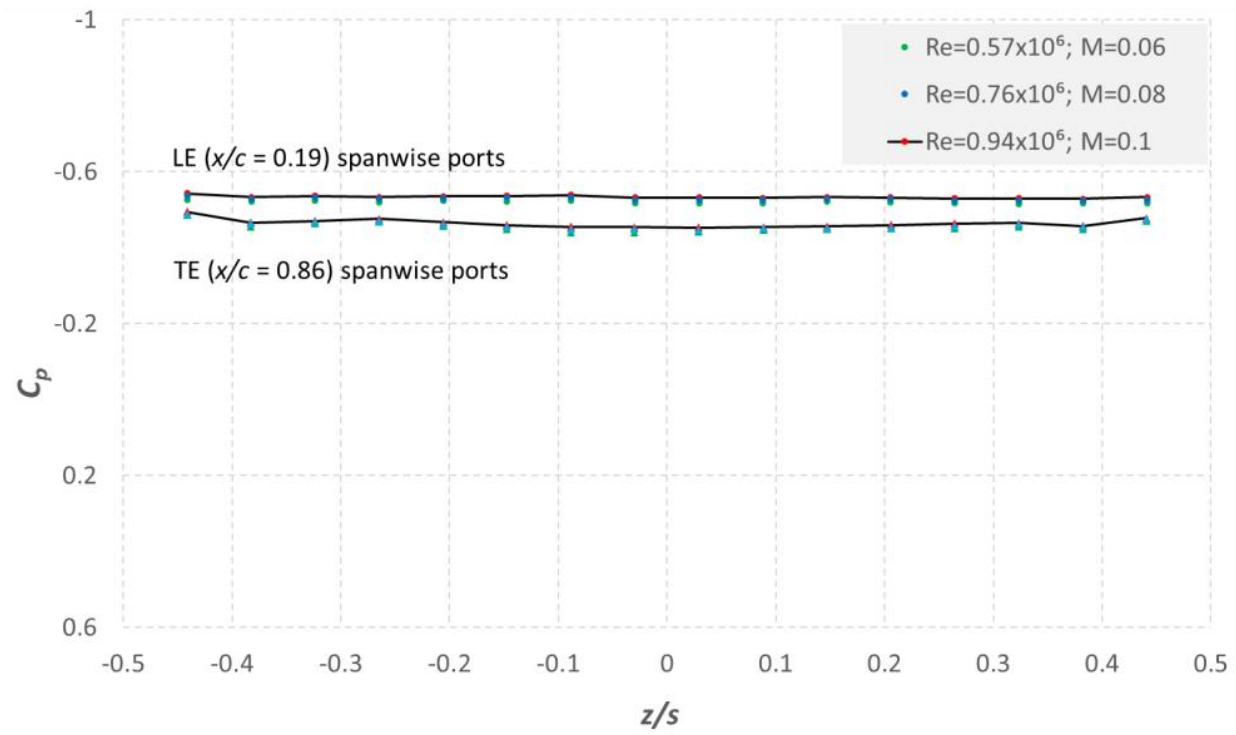

Figure 9. The effect of Reynolds number on the spanwise flow uniformity for the baseline case. 


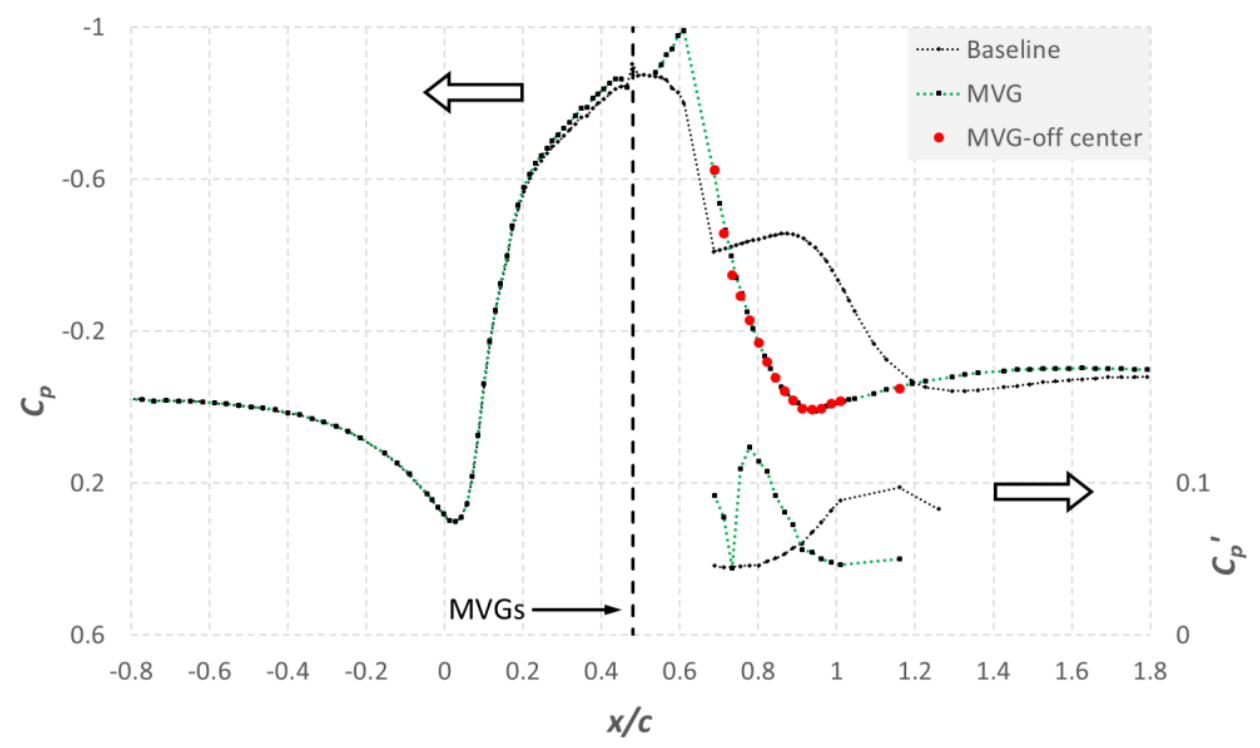

Figure 10. Surface pressure distributions for the MVG flow control case.

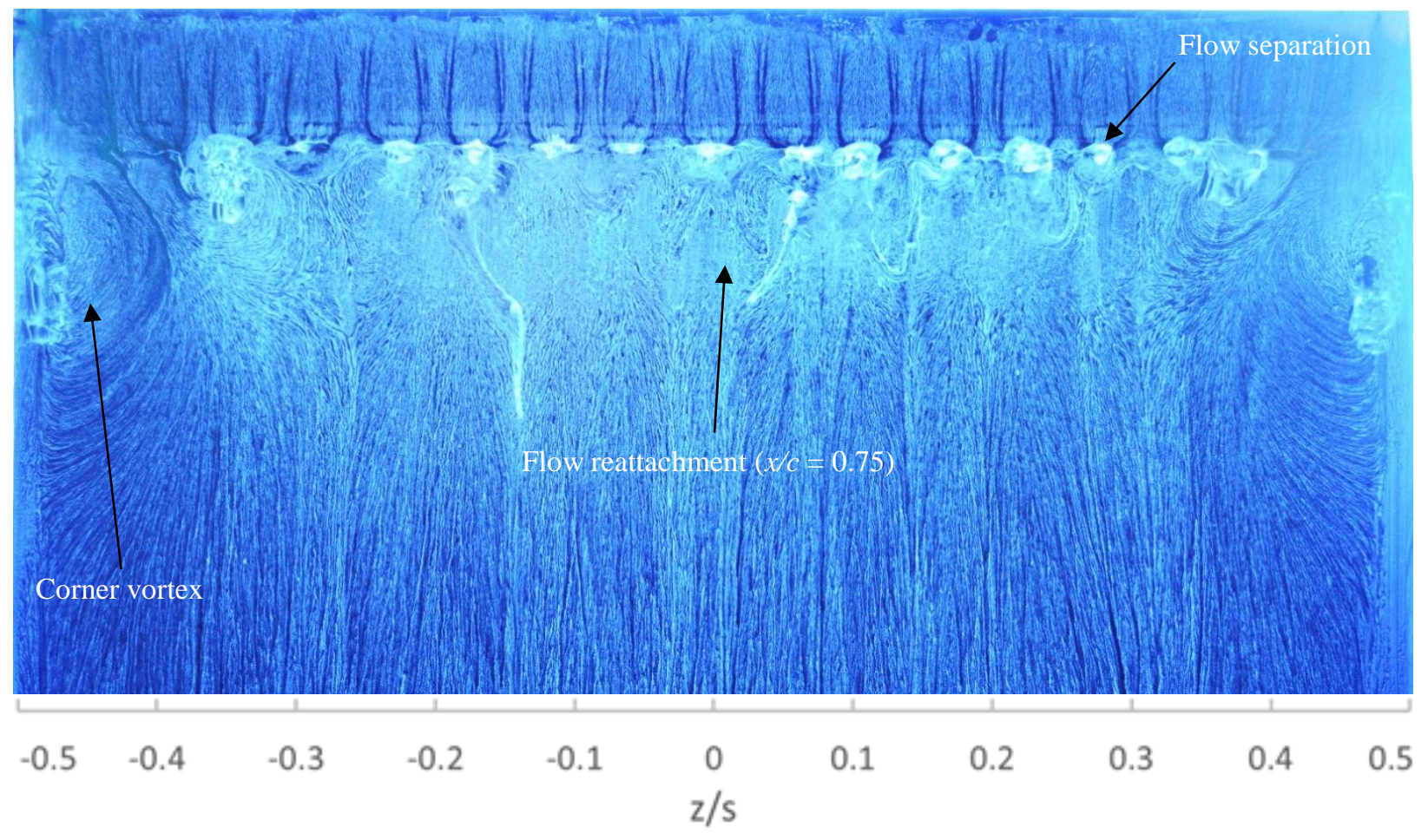

Figure 11. Surface oilflow visualization for the MVG flow control case. 


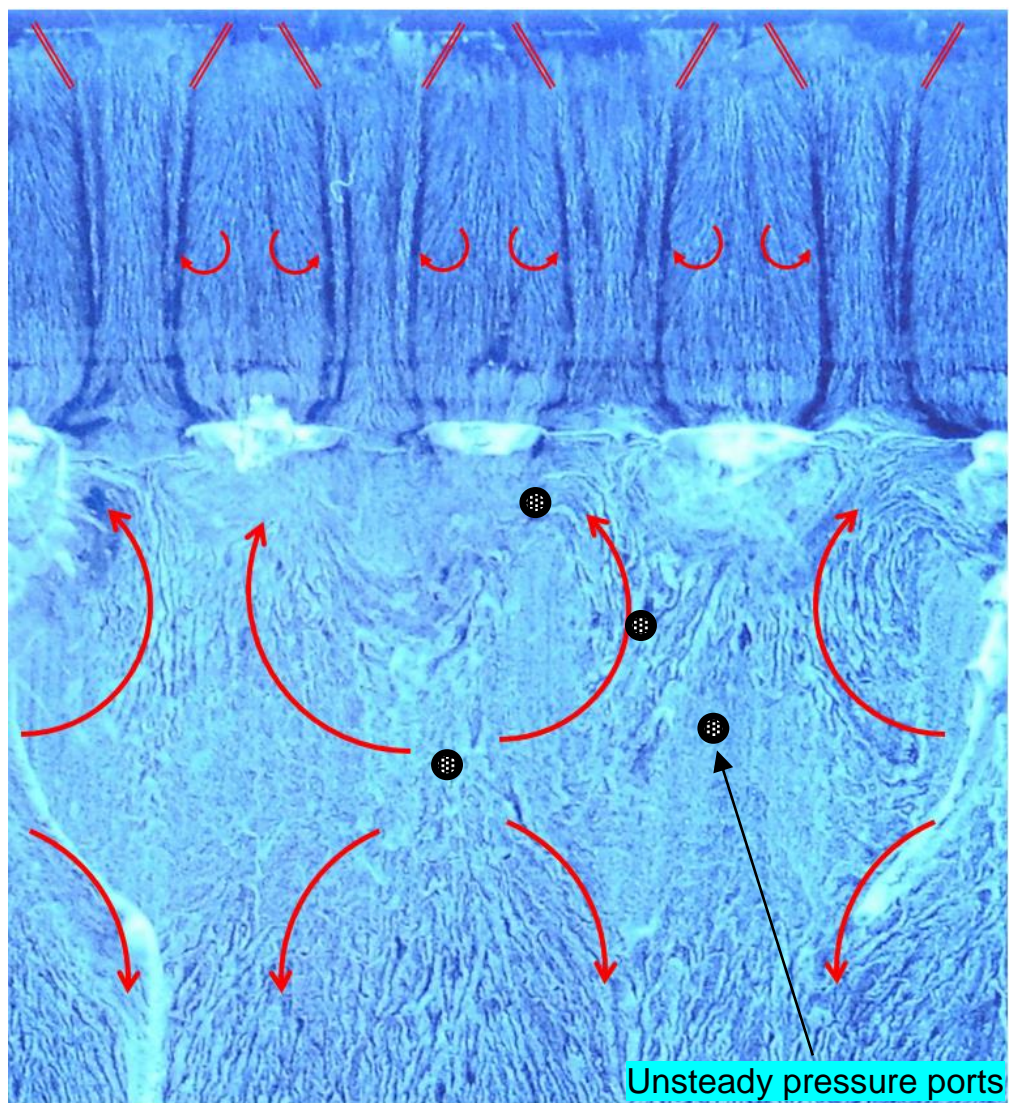

Figure 12. Zoomed-in image of oilflow visualization for the MVG flow control case.

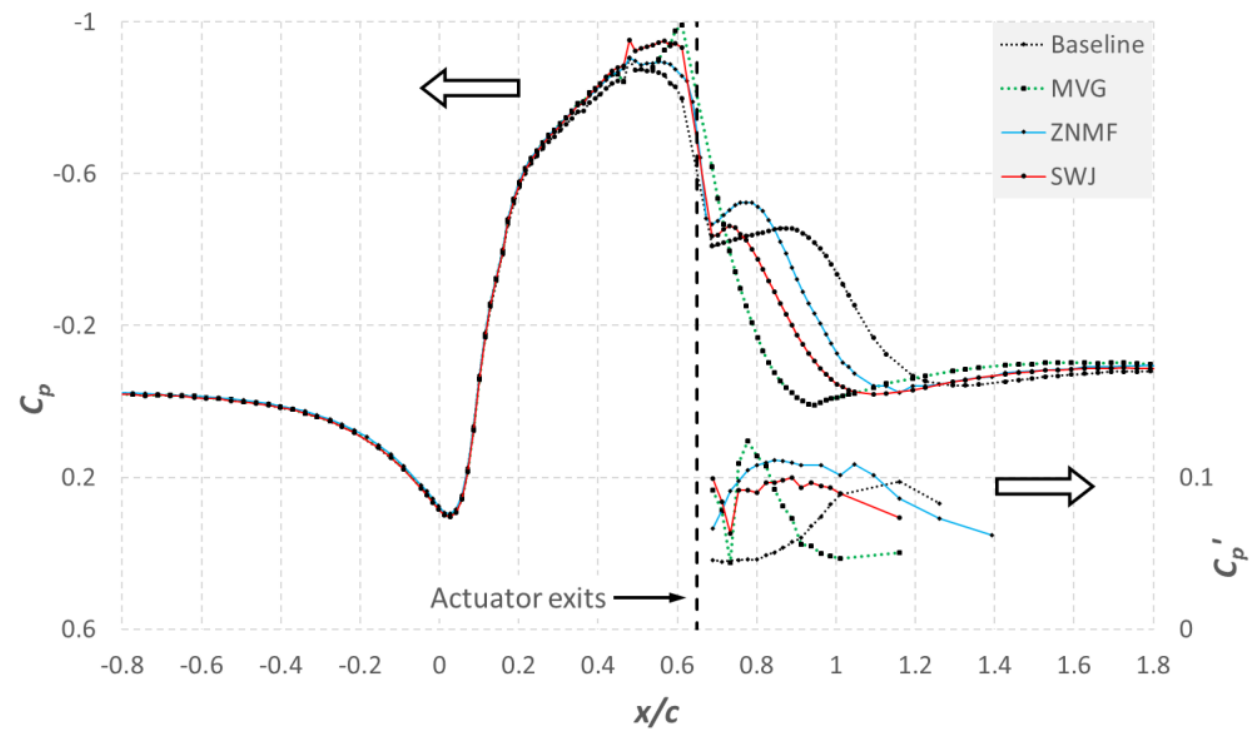

Figure 13. Comparison of the SWJ and $\mathrm{ZNMF}^{12}$ flow control cases for $C_{\mu}=0.11 \%$. 


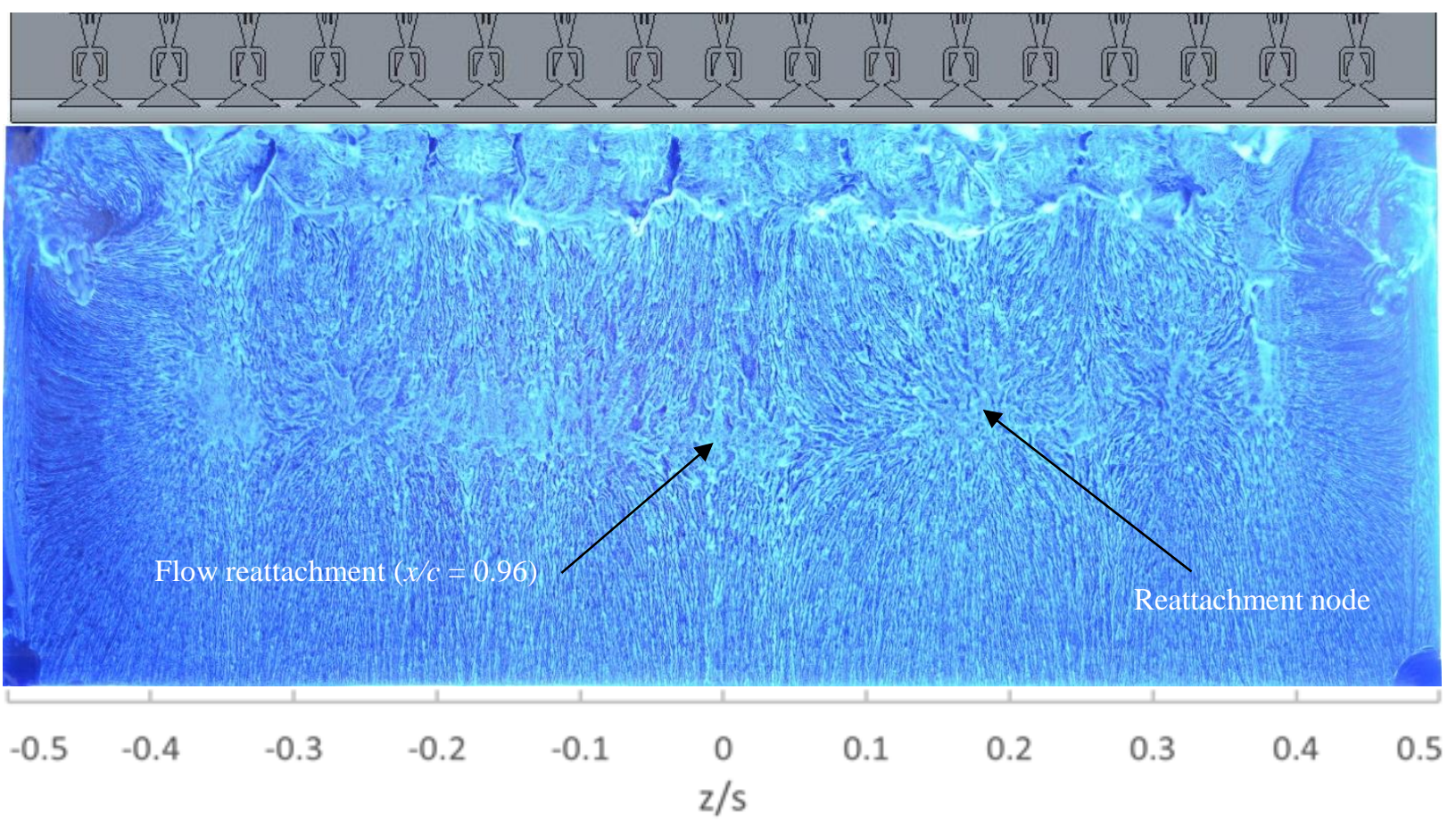

Figure 14. Surface oilfow visualization for the SWJ flow control case with $C_{\mu}=0.11 \%$.

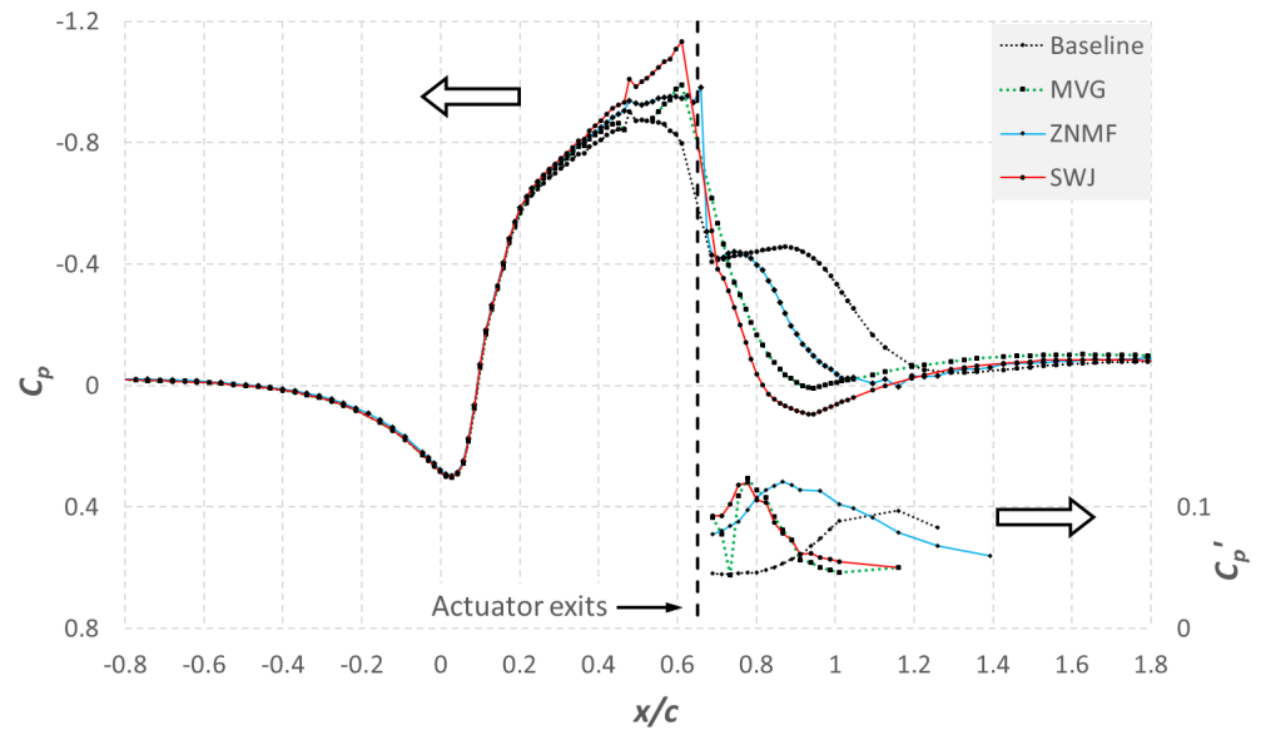

Figure 15. Comparison of the SWJ and $\mathrm{ZNMF}^{12}$ flow control cases for $C_{\mu}=0.35 \%$. 


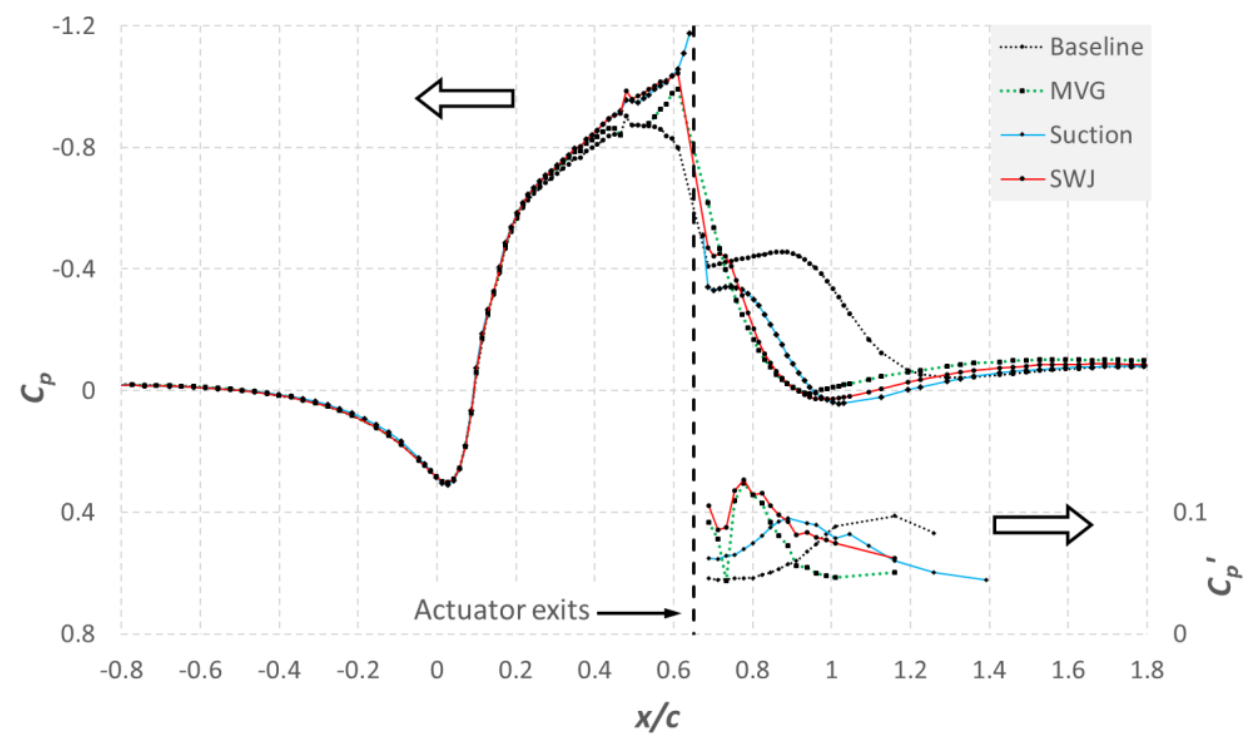

Figure 16. Comparison of the SWJ actuator with steady suction ${ }^{11}$ for $C_{\mu}=0.24 \%$.

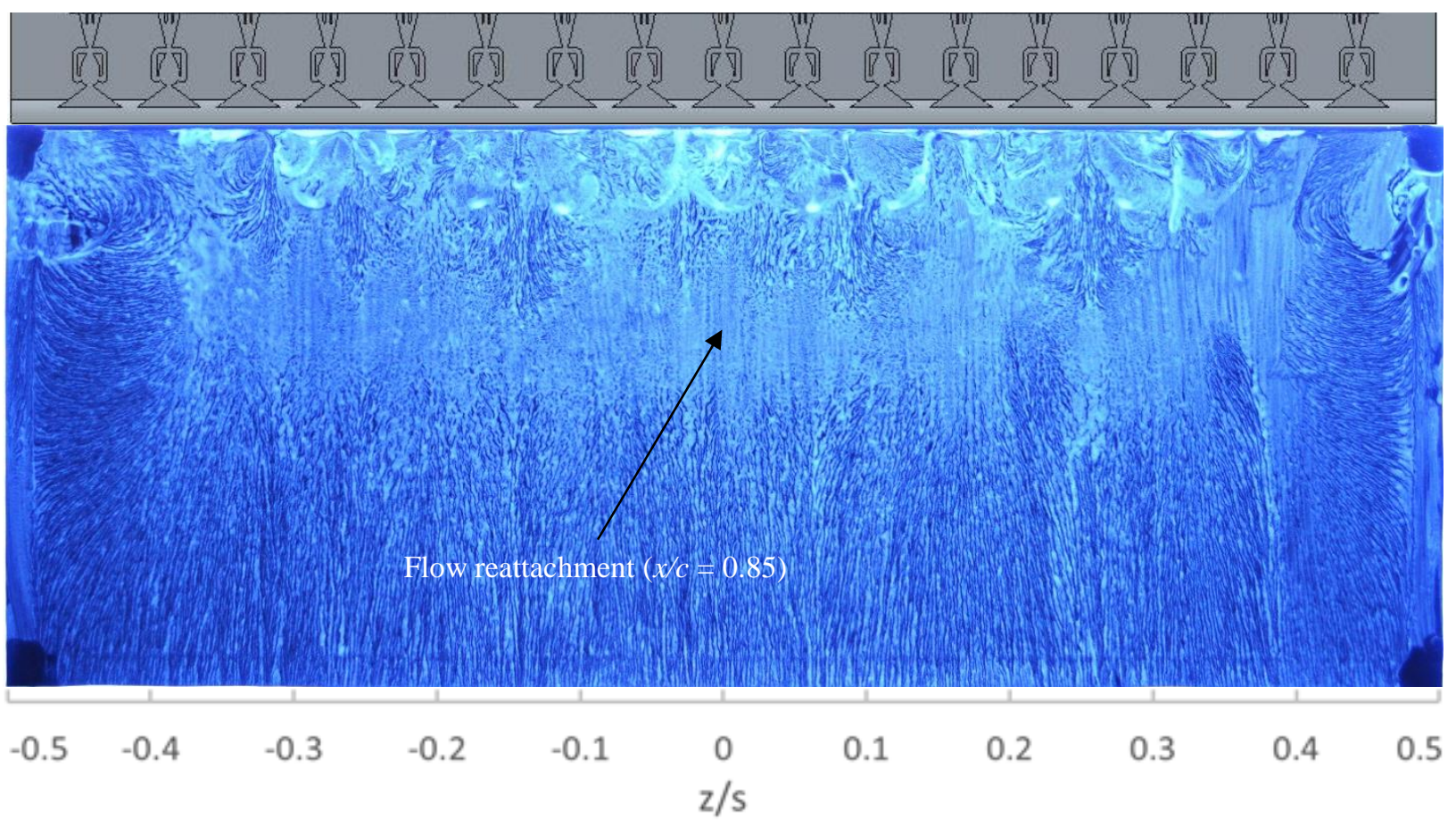

Figure 17. Surface oilfow visualization for the SWJ flow control case with $C_{\mu}=0.24 \%$. 


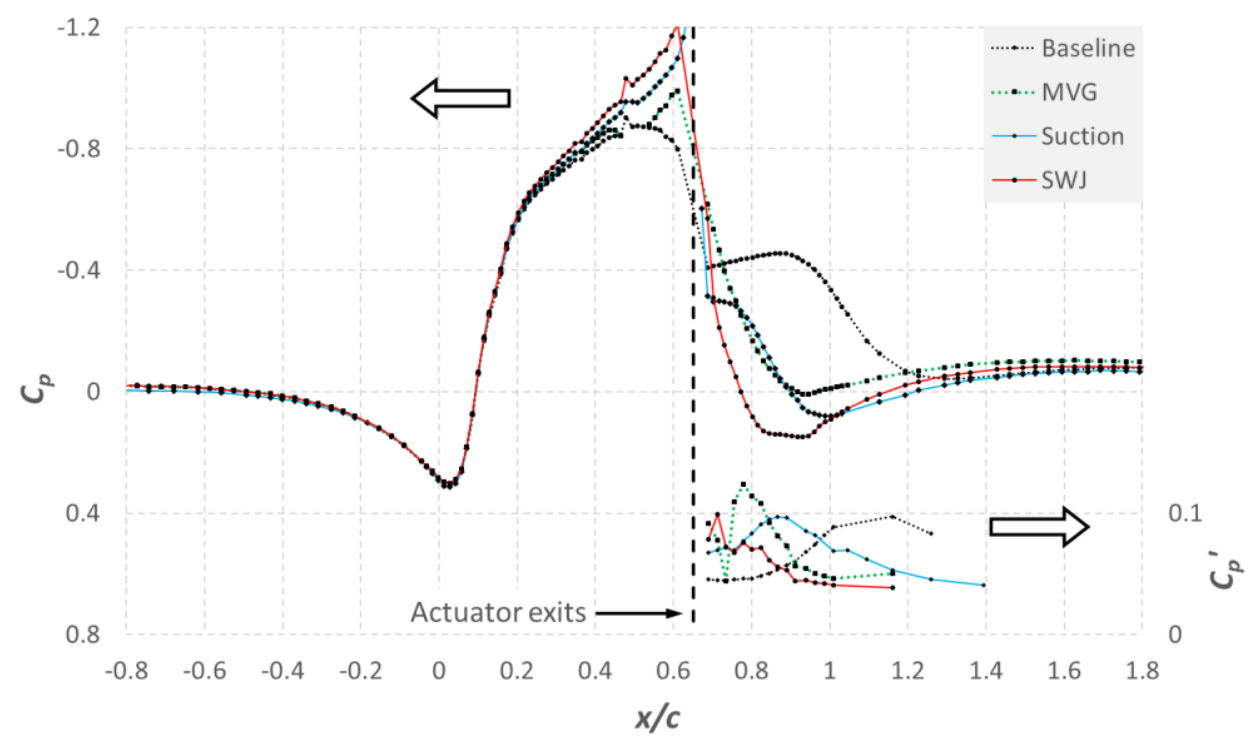

Figure 18. Comparison of the SWJ actuator with steady suction ${ }^{11}$ for $C_{\mu}=0.47 \%$.

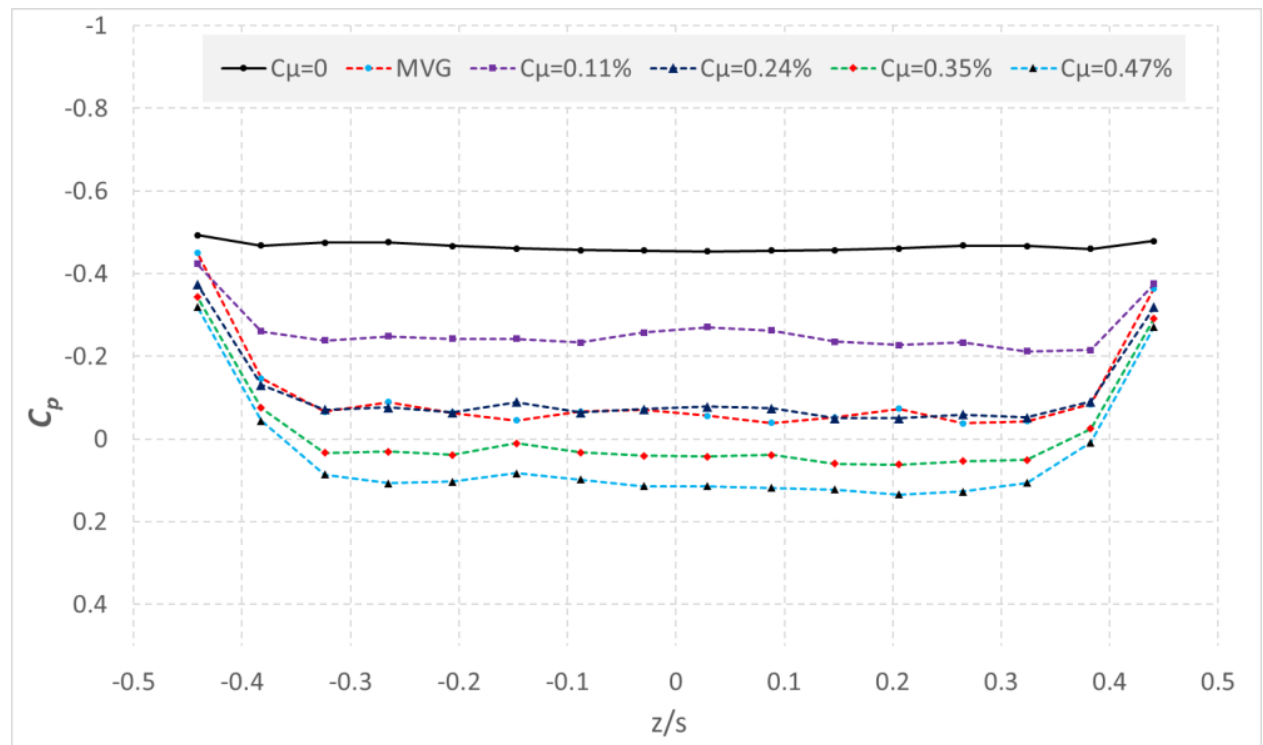

Figure 19. Spanwise pressure distribution for various level of SWJ excitation at $x / c=0.86$. 